\title{
Les « territoires » stylistiques du premier Mésolithique en Poitou-Charentes et Pays-de-la- Loire
}

Sylvène Michel

\section{CpenEdition}

\section{Journals}

Édition électronique

URL : http://journals.openedition.org/rao/234

DOI : $10.4000 /$ rao. 234

ISBN : 978-2-7535-1607-6

ISSN : 1775-3732

Éditeur

Presses universitaires de Rennes

\section{Édition imprimée}

Date de publication : 30 décembre 2007

Pagination : 7-30

ISBN : 978-2-7535-0574-2

ISSN : 0767-709X

Référence électronique

Sylvène Michel, «Les «territoires » stylistiques du premier Mésolithique en Poitou-Charentes et Paysde-la-Loire », Revue archéologique de l'Ouest [En ligne], 24 | 2007, mis en ligne le 30 décembre 2009, consulté le 06 décembre 2020. URL : http://journals.openedition.org/rao/234 ; DOI : https://doi.org/ $10.4000 /$ rao. 234 


\title{
Les « territoires" stylistiques du premier Mésolithique en Poitou-Charentes et Pays-de-la-Loire ${ }^{1}$
}

\author{
Sylvène MiCHEL*
}

\begin{abstract}
Résumé : La faible connaissance des groupes humains du premier Mésolithique dans le centre-ouest de la France découle de rares travaux, souvent centrés sur des zones géographiques autres et ne s’appuyant que sur quelques sites. L'examen suivant, basé sur une approche stylistique des territoires via l'étude des armatures, prend en compte l'ensemble des cas répondant aux critères d'étude qui ont pu être recensés. Le modèle proposé se démarque de certains schémas et n’intègre pas totalement le premier Mésolithique du Centre-Ouest à la sphère sauveterrienne : il repose sur des éseaux d'interactions croisées entre différents faciès, dont ceux de Loire-Atlantique/Vendée (Mésolithique ancien ligérien) et des Charentes (Mésolithique ancien charentais).
\end{abstract}

\begin{abstract}
The First Mesolithic style "territories" in Poitou-Charentes and Pays-de-la-Loire (Central-Western France)
The little we know of the first Mesolithic human groups of the french Centre-Ouest derived from rare discoveries, often focusing on other geographical areas and relying on only a few sites. The following examination, based on a stylistic approach to territories via the study of arrowheads, takes into account the whole range of cases, from which we were able to make an inventory which fulfilled the criteria of the study. The model suggested doesn't include completely the early Mesolithic of Central Western France in the Sauveterrian sphere and is based on networks of cross-interactions between different facies, notably those of Loire-Atlantique/Vendée (Early Ligerian Mesolithic) and Charentes (Early Charentais Mesolithic).
\end{abstract}

Mots clés : Premier Mésolithique, Centre-Ouest, armatures, territoires stylistiques.

Key words: First Mesolithic, Center-West France, arrowheads, stylistic territories.

Les études concernant les productions lithiques des premiers groupes humains de l'Holocène du centre-ouest de la France ${ }^{2}$ connaissent un réel dynamisme depuis une vingtaine d'années, notamment dans le Sud-nantais, le bocage vendéen ainsi que dans les vallées de la Vienne et de la Charente (fig. 1). Cependant, la rareté des synthèses, la défaillance des cadres conceptuels et chrono-stratigraphiques ainsi que le nombre important de collections de prospections inédites ont pour conséquence une mauvaise appréhension des peuplements en Poitou-Charentes et Pays-de-la-Loire entre les X ${ }^{e}$ et VII ${ }^{e}$ millénaires cal. B.C. (Gouraud, 1995;
1996; Marchand et al., 1998; Gouraud et Marchand, 1999; Foucher et San Juan, 2004). L'étude suivante propose, pour participer au renouvellement des caractérisations des entités culturelles en jeu, de se concentrer plus particulièrement sur la problématique des territoires. Une réflexion sur les notions d'identité et de territoire, ainsi que sur l'évaluation de leur transcription dans le monde matériel, semble nécessaire pour mener avec pertinence un tel questionnement. Dans ce travail liminaire, l'analyse de l'organisation des territoires stylistiques se base principalement sur une étude typologique des armatures, outils les plus affectés par les variations

* Doctorante, UMR 6566 du CNRS - université de Rennes 1, 263 avenue du général Leclerc, 35042 Rennes Cedex 1.

1. Cet article reprend les données d'une étude réalisée en 2005/2006 dans le cadre d'un diplôme de Master 2 (spécialité Archéologie et Archéosciences : Préhistoire et Protohistoire à l'université de Rennes 1 sous la direction de Grégor Marchand [UMR 6566]). Je remercie ceux qui, comme F. Blanchet (Service régional de l'Archéologie de Poitou-Charentes), P. Fouéré (INRAP - UMR 5808 - Institut de Préhistoire et Géologie du Quaternaire), E. George (Carte archéologique Pays-de-la-Loire), M. Mazière (Carte Archéologique Poitou-Charentes), m’ont aidée à récolter et traiter les informations concernant ce sujet.

2. Dans l'étude suivante, le terme Centre-Ouest désigne les régions Pays-de-la-Loire et Poitou-Charentes. Le Grand-Ouest correspond à la zone englobant la façade atlantique de la Basse-Normandie à l'Aquitaine, sur une profondeur d'environ $300 \mathrm{~km}$ vers l'est (fig. 1). 


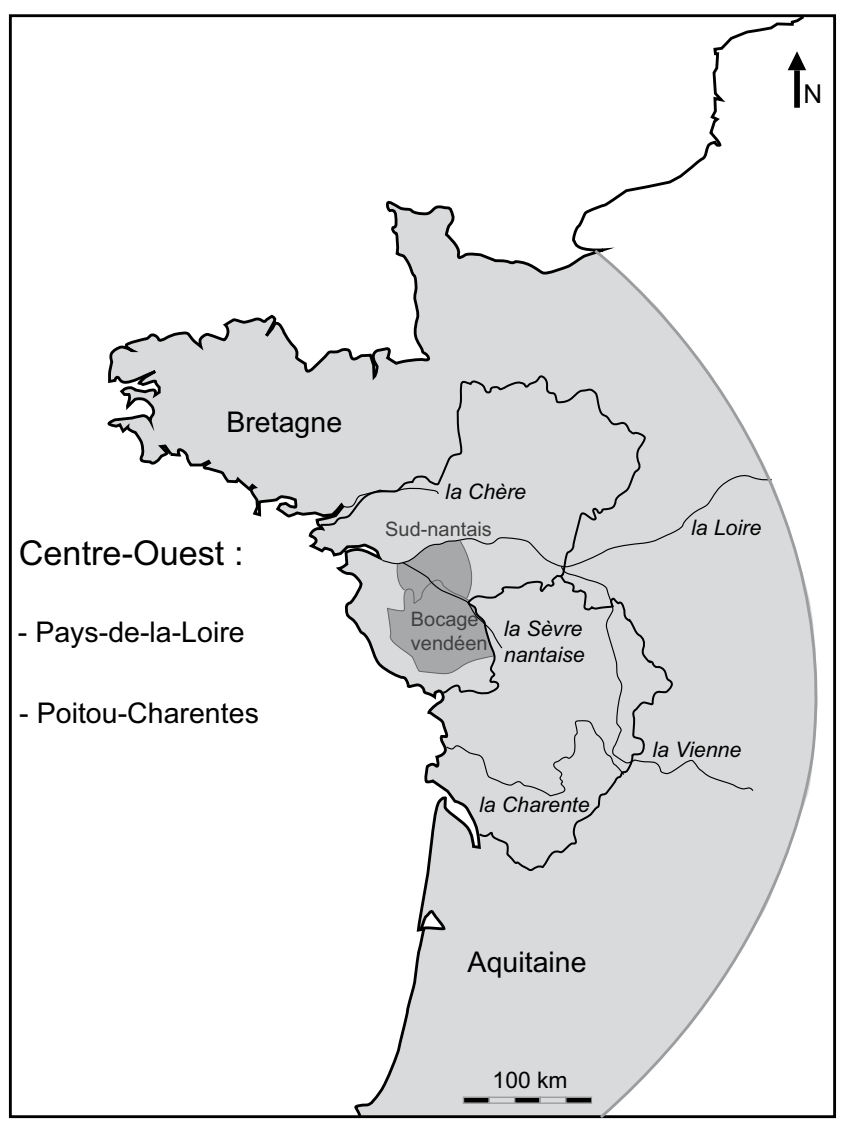

Figure 1 : Localisation du cadre d'étude : zones géographiques et cours d'eau mentionnés dans le texte.

Figure 1: Location of the study area, with geographical zones and rivers mentionned in text.

culturelles, et sur un examen de leur répartition spatiale avec un Système d'Information géographique (SIG).

\section{ENTRE Loire ET GARONNE : DÉFINITION CULTURELLE}

L'expression " premier Mésolithique » désigne ici les phases ancienne et moyenne de cette période, de 9700-8020 cal. B.C. à 8020-6900 cal. B.C. (Cupillard et Richard, 1998). En ce début d'Holocène, parallèlement à un réchauffement climatique à l'origine de la mise en place d'une forêt de pins où petit à petit noisetiers, ormes ou tilleuls s'épanouissent et d'une faune où cerfs et sangliers dominent, l'outillage connaît une évolution importante avec un phénomène de microlithisation généralisé. Des lamelles irrégulières et sinueuses, issues de débitage de type Coincy, servent de support pour façonner des armatures géométriques (triangles, segments) ou non : pointes, lamelles à bord(s) abattu(s).

Les travaux d'identification des groupes culturels du Centre-Ouest ne dérogent pas aux méthodes développées ailleurs en Europe puisqu'ils reposent sur une étude préférentielle de ces microlithes. Cette zone géographique n'a souvent été abordée qu'en filigrane à travers les études concernant les régions avoisinantes. Les résultats obtenus varient. Certains modèles ne définissent pas de groupe stylistique pour les Pays-de-la-Loire et/ou le Poitou-Charentes au Mésolithique ancien et/ou moyen (Kozlowski, 1975; 2003; Rozoy, 1997; Ducrocq, 2001), invoquant souvent une insuffisance qualitative et quantitative des études. Ceux qui proposent des divisions culturelles posent parfois certains problèmes : les « ethnonymes " (Boissinot, 1998) employés correspondent rarement à une définition clairement énoncée et, lorsque les critères de définition sont explicités, ils semblent souvent insuffisants à eux seuls pour être utilisés comme les bases d'une culture. Le recours à un trop faible nombre de types référents - voire à un seul - pour définir un groupe stylistique, ou l'appartenance à ce groupe, est par exemple difficilement soutenable. Bien que l'abandon du concept de "fossile directeur " au profit de caractérisations par association de différents types d'armatures soit prôné depuis plusieurs décennies (Rozoy 1978b), la mise en application de cette idée demeure encore trop rare. De plus, les " ethnonymes » caractérisés sont parfois détournés de leur sens originel ou desservis par des qualitatifs vagues, telle la très récurrente expression "Sauveterrien au sens large ", ne faisant alors plus appel à une signification réelle.

L'approche des territoires du Centre-Ouest ne semble donc pas toujours adaptée, faute notamment de cadre conceptuel clair. Établir une synthèse sur le sujet s'avère d'autant plus complexe que les propositions de divisions culturelles sont variées, voire en partie contradictoires. Ainsi, quelques précisions et révisions paraissent nécessaires, notamment en ce qui concerne l'« extension » et l'« impact » du Sauveterrien souvent évoqués pour les régions présentement étudiées; les réflexions concernant un techno-complexe sauveterrien central influençant "sa périphérie » sont à revoir (cf. infra).

\section{Territoires du Premier MésolithiQue en Centre-Ouest : NOUVElles PERSPECTIVES}

La démarche suivante, au risque de paraitre restrictive, répond à une volonté de clarifier certaines définitions; elle comporte ainsi un certain nombre d'éléments qui restent largement perfectibles.

\section{Entité culturelle et territoire?}

Vouloir définir de manière claire les concepts d'identité et de groupe culturel s'avère être une tâche ardue étant donné les approches divergentes des différentes disciplines 
et écoles. Un certain consensus semble établi sur le fait que l'identité désigne la conception qu'a l'individu de lui-même et de l'environnement social auquel il a le sentiment d'appartenir (Brunet et al., 1992). Elle s'exprime à travers le groupe, qui n'en est qu'une catégorie analytique et subjective (Giddens, 1987). Fondé par l'inconscient collectif et suivant des normes sociales, celui-ci est caractérisé par un ensemble d'éléments discriminants basés sur des facteurs sociaux, linguistiques, démographiques, psychiques, symboliques, idéologiques, ou économiques. Au sein du débat qui anime les sciences humaines, le concept de culture semble problématique. Traditionnellement, l'archéologue associe les cultures matérielles - incarnées par les vestiges découverts - à la culture, ensemble complexe des comportements et savoirfaire à l'origine de ces vestiges. Pour certains chercheurs, ce point de vue ne respecte pas la définition de culture comme système en perpétuelle évolution (Barth, 1969; Bonte et Izard, 1991). Ils remettent alors plus ou moins en question la possibilité d'identifier et de catégoriser des entités humaines à partir d'objets (Boissinot, 1998) qui ne reflètent que certains des sous-systèmes de leur culture, d'autres encore pouvant même ne pas laisser de traces appréciables par l'archéologue. La caractérisation des groupes humains passés est alors également considérée comme indissociable des raisonnements culturalistes délimitant des ensembles clos (Demars, 1986; Olivier et al., 2000; Lemercier, 2000).

Certains points soulignés par ces critiques sont justifiés. Cependant, pour ne pas rester dans le simple fait descriptif, le préhistorien n'a pas d'autre alternative que de reconstituer des aspects non matériels d'un groupe humain - telle l'organisation sociale, culturelle et cultuelle - à partir des seules données qui sont à sa disposition. Une des méthodes principales pour, non pas appréhender totalement les comportements des groupes de chasseurs-cueilleurs mais les évaluer au mieux, reste actuellement l'analyse détaillée de leur industrie osseuse mais surtout lithique. Certes, elle ne constitue qu'une facette de la culture matérielle originelle, mais quel que soit le type d'indices à disposition, la communauté observée ne pourra être appréhendée que de manière partielle. Ces entités matérielles doivent donc être traitées le plus pertinemment possible, sans perdre de vue qu'il ne faut pas projeter strictement leurs résultats en terme de mode de vie et de pensée, au risque de tomber dans la surinterprétation.

La notion de territoire est difficilement séparable de celle de groupe culturel, aussi bien pour les peuples sédentaires que nomades. En effet, ces derniers ne peuvent pas être considérés comme dépourvus de système spatial structuré et d'attachement culturel ou symbolique au substrat géographique (Yven, 2004). Le territoire résulte d'une construction sociale évolutive à partir d'un paysage naturel. Cet espace approprié par le groupe (Bonte et Izard, 1991) est structuré, en fonction de besoins précis, par des réseaux connectant différents pôles. L'occupation de l'espace par une communauté humaine, ou sa zone de parcours, est contrainte par des facteurs qui lui sont externes ou internes. Ses frontières, qu'il ne faut pas réduire à un simple tracé sans volume, peuvent donc être naturelles mais aussi mentales. L'ethnologie a souvent montré qu'un groupe humain investit une surface choisie et non pas la totalité de l'espace dont il dispose (Collignon, 1996). L'héritage et la mémorisation du territoire des ancêtres jouent un rôle pour ce qui concerne la seconde catégorie de frontières énoncée (Gouletquer, 1979).

L'observation de la répartition spatiale d'éléments discriminants permet de contribuer à l'identification des territoires suivant différents niveaux de perception. Leur nature varie en fonction de l'élément référent choisi qui peut offrir des informations sur l'histoire culturelle et technique d'un groupe donné, ou sur son territoire de parcours associé notamment à l'acquisition des matières premières. Leur interaction forme un reflet des territoires identitaires et humains, support de l'identité collective. L'entité spatiale dessinée par les traceurs territoriaux de type matériel ne correspond donc pas obligatoirement en totalité à celles qui pourraient être déterminées à partir d'autres aspects de la culture. Mais, pour des raisons déjà énoncées, ceci n'est en rien une raison de s'abstenir d'utiliser cette méthode; seule importe la modulation de l'interprétation.

Les remarques et critiques précédentes soulignent le besoin de s'interroger sur les notions clés de la palette de l'archéologue que sont l'identité culturelle, sa transcription dans le monde matériel et sa représentation dans les territoires.

\section{Marqueurs culturels}

Le marqueur jusqu'alors privilégié dans l'identification de territoires stylistiques mésolithiques est l'armature, « facteur d'identité [...] d'un groupe culturel ou de groupes apparentés " (Thévenin, 1999), à travers la déclinaison de types. Ce choix appelle la notion de style matériel, dont la définition est à ouvrir au-delà de la simple combinaison d'attributs morphologiques en y intégrant également l'aspect technologique et fonctionnel (Dietler et Herbich, 1994). Le style s'appréhende alors comme le résultat d'une gamme caractéristique de choix interdépendants effectués à tous moments de la chaîne opératoire de production (ibid.). Les observations ethnologiques montrent que la combinaison des facteurs définissant des types d'armatures - au niveau de la pointe de flèche mais aussi de l'empennage et de la hampe - contribue à assurer l'identité culturelle (Lemonnier, 1987; Pétrequin et Pétrequin, 1990). Elles en soulignent par là même la " richesse sémantique " (Giligny et Sidi-Maamar, 1990), cette nature polysémique des armatures s'exprimant 
à travers des paramètres techniques, fonctionnels, sociaux, symboliques ou proprement culturels, tout en prenant en compte le phénomène d'idiosyncrasie. Certes les microlithes n'offrent que des reflets, partiellement déformés, des cultures qui les sous-tendent. Il n'en reste pas moins que c'est actuellement à partir de ces éléments, qui participent activement à la vie quotidienne des chasseurs-cueilleurs, que nous sommes les plus aptes à détecter et caractériser les cultures mésolithiques. L'analyse des armatures requiert une certaine précaution dans l'interprétation. En effet, les microlithes ont connu un discrédit dans l'observation des groupes identitaires (Boissinot, 1998), dû à leur exploitation stylistique accrue ayant occasionné des déductions sociales ou culturelles trop exclusives, avec au final pour effet pervers un certain ralentissement des études typologiques. Ainsi, le recours à un unique type d'armature pour caractériser un groupe identitaire (cf. supra) n'apparait pas réellement pertinent : seule une association de types permet d'aboutir à cette restitution. Le rapport quantitatif entre outillage commun et armatures ne semble quant à lui plus être appréhendé comme un indice culturel, mais traduirait la nature des activités menées (Valdeyron in Barbaza et al., 1991; Marchand, 2000).

La typologie proposée s'appuie sur les critères utilisés dans les travaux de référence sur les armatures mésolithiques (GEEM, 1969; 1972; Rozoy, 1978a ; Soler Garcia, 1982; Gouraud, 1987; Valdeyron, 1994; Gouraud et Marchand, 1999). Ces caractères ont été complétés et réorganisés dans l'optique de valoriser l'association de la typologie à la technologie. Le système de codification proposé repose sur des critères concrets structurés en quatre niveaux agencés en fonction d'aspects technologiques (fig. 2). Cette approche vise à définir au plus près le champ des possibles, d'où de nombreux sous-types, puis à identifier les choix préférentiels des tailleurs concernés. Afin d'éclaircir nos propos, une correspondance a été établie par la suite entre la codification brute et les appellations déjà en vigueur, en respectant des définitions strictes (tabl. 1).

La difficulté d'attribuer certaines pièces à un type est parfois réelle et s'accroît pour les pièces fragmentées. C'est pourquoi, afin de ne pas fausser les répartitions des types d'armatures, nous nous sommes abstenus de les identifier en cas de doute. L'ensemble des caractères choisis pour les pointes à base retouchée, même ceux jugés " discrets ", s'est avéré pertinent pour le repérage d'armatures locales et donc pour la mise au jour de groupes stylistiques (cf. infra). Ces critères ne se combinent pas pour autant tous entre eux, ce qui explique que seule la moitié des possibilités proposées pour ce type a effectivement été réalisée. Évoquons enfin le cas des armatures en cours de fabrication : elles méritent une étude typo-technologique détaillée et normalisée compte

\begin{tabular}{|c|c|c|c|c|}
\hline Appellation & $\begin{array}{c}\text { Pointe de la } \\
\text { Majoire }\end{array}$ & $\begin{array}{c}\text { Pointe de } \\
\text { Chaville }\end{array}$ & $\begin{array}{c}\text { Pointe de } \\
\text { Sauveterre }\end{array}$ & $\begin{array}{c}\text { Triangle de } \\
\text { Montclus }\end{array}$ \\
\hline Codification & 72.2 & 12.1 & 71.2 & $3 . \ldots 2 ; 6.22$ \\
\hline
\end{tabular}

Tableau 1 : Principaux exemples de codification utilisés. Il faut y rajouter les critères dimensionnels. La retouche du triangle de Montclus peut se faire suivant le principe de la troncature uniquement (3...2) ou associer ce dernier, pour le petit côté, au principe du bord abattu, ce qui revient à abattre une lamelle et la tronquer (6.22).

Table 1: Principal examples of the coding employed (metrical information excluded).

tenu de leur fort pouvoir informatif. Les études lithiques à venir en Centre-Ouest et dans les zones alentour permettront d'affiner cette proposition typologique.

Les territoires stylistiques mésolithiques jusqu'alors modélisés à partir de l'observation des types d'armatures reposent sur des tendances générales. Un renouvellement de cet axe de recherche est proposé ici grâce à l'utilisation d'un Système d'Information Géographique (SIG) dans l'analyse des marqueurs typologiques. À la précision de la représentation cartographique s'ajoute un meilleur suivi de l'organisation des territoires grâce à un croisement des diverses données les concernant. L'étude qui suit s'est appuyée sur une observation des proportions et distributions des (sous-)types d'armatures et de leurs combinaisons, le but étant de voir si des agencements morphologiques non repérés à l'échelle d'un site se dégageaient à l'échelle régionale. Tous ces résultats ont été traités avec la prudence que l'usage des statistiques requiert, notamment en raison des contraintes du phénomène d'échantillonnage.

\section{Exploitation de l'information}

L'ensemble des indices du premier Mésolithique connus a été recensé ${ }^{3}$, y compris les traces ténues, parfois isolées au sein d'industries non contemporaines (fig. 3; tabl. 2). Pour l'étude des territoires, seules ont été prises en compte les collections pour lesquelles la bibliographie offrait des descriptions typologiques des armatures à travers commentaires écrits et des-

3. Cet inventaire se base sur le dépouillement de revues nationales et locales: Bulletin de la Société préhistorique française, Revue archéologique de l'Ouest, Bulletins mensuels et Études de la Société nantaise de Préhistoire, Groupe vendéen d'Études préhistoriques, Société d'Émulation de la Vendée, Bulletin de la Société d'Agriculture, des Sciences et des Arts de Sarthe, Bulletin de la Société d'Études scientifiques de l'Anjou, La Province du Maine, La Mayenne: Archéologie et Histoire, Bulletin de la Commission historique et archéologique de la Mayenne, Études préhistoriques et protohistoriques des Pays-de-la-Loire, Bulletins et Mémoires de la Société archéologique et historique de la Charente, Bulletin de la Société d'Archéologie et d'Histoire de Charente-Maritime, Roccafortis, Revue des Provinces de l'Ouest (Bretagne, Poitou, Anjou). Il s'appuie aussi sur la consultation de rapports de fouilles/prospections et de monographies, l'interrogation des Cartes archéologiques des régions concernées, ainsi que sur des données inédites transmises par F. Blanchet. 


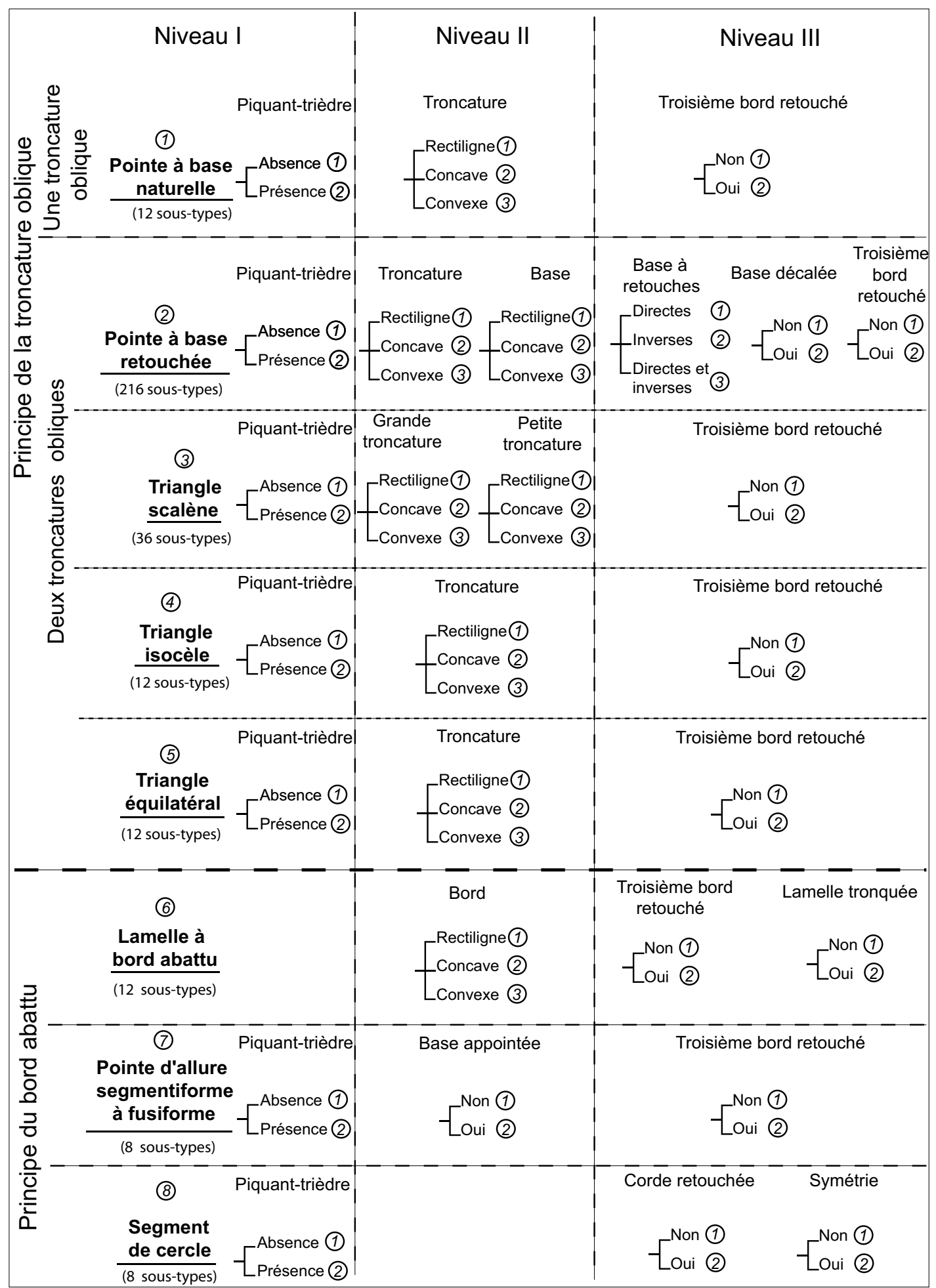

Figure 2 : Typologie adoptée dans la présente étude. Chiffres arabes, système de codification; chiffres romains,: niveaux typologiques: I, mode de fabrication de l'armature et critères techniques; II, phase de retouche; III, caractères « discrets ». Le niveau IV concerne les dimensions de l'armature; leur mesure n'a pas été codée mais conservée telle quelle, ainsi ce niveau n'est pas représenté sur ce schéma; il n’en reste pas moins primordial pour la définition des types..

Figure 2: Typology used here. Arabic figures for coding system; Roman figures for typological sets: I, arrowhead fabrication and technical criteria; II, retouch; III, "discret" criteria (Set IV concerns arrowhead dimensions, not coded here). 


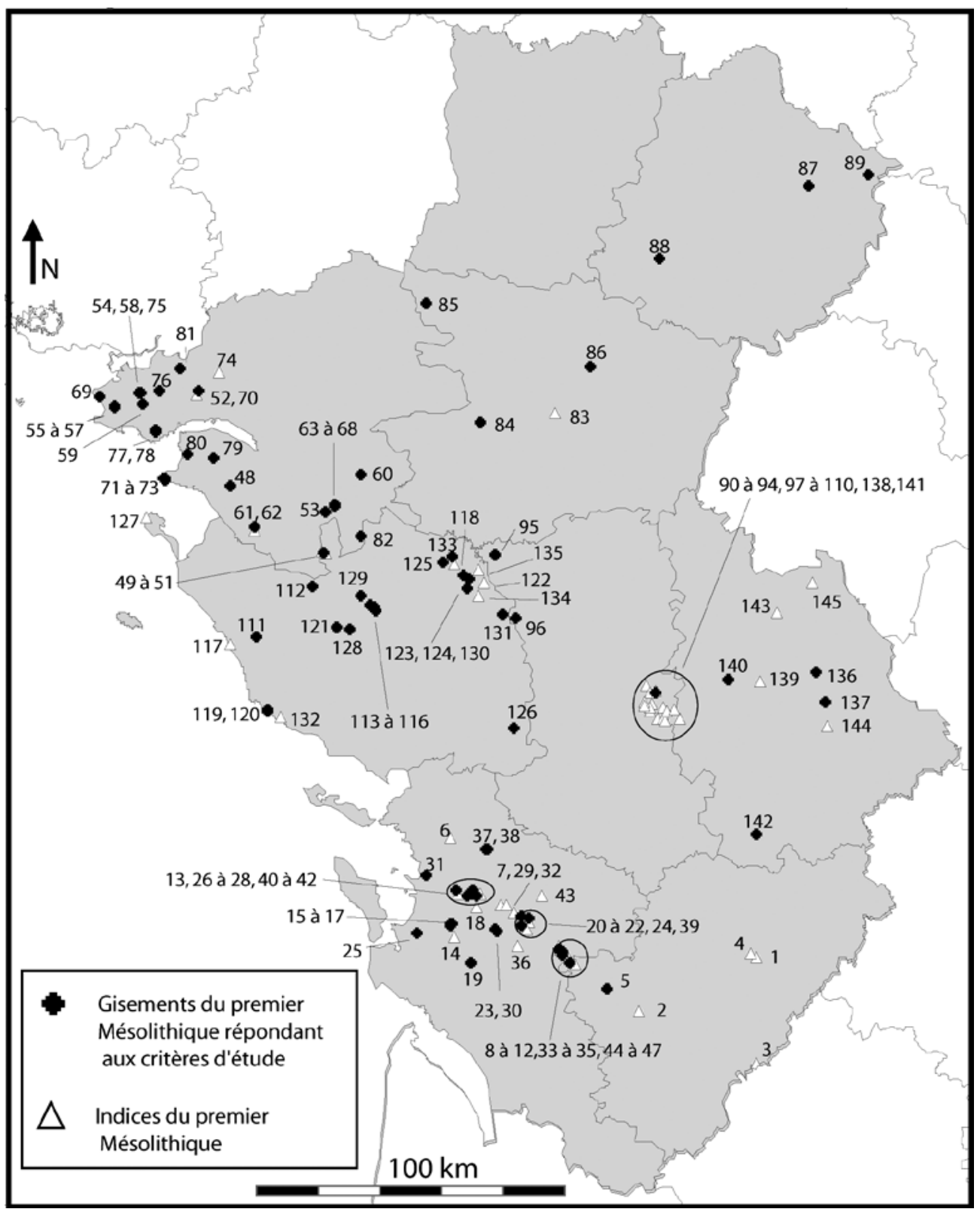

Figure 3 : Localisation des gisements et indices du premier Mésolithique recensés ; les chiffres renvoient au tableau 2 (DAO : S. Michel).

Figure 3: Location of the studied early Mesolithic sites and indices (figures refer to Table 2).

sins. La présence de documents iconographiques était une condition principale pour homogénéiser les données - consignées et dessinées par des classificateurs d'horizons, d'époques et de formations variés - et vérifier la validité de certaines informations. En effet, les attributions typologiques étaient parfois imprécises ou à reprendre : ainsi, quelques pointes à l'extrémité apicale cassée avaient été associées à des pointes de la Majoire ou de Chaville, classes typologiques nécessitant la présence d'un piquant-trièdre. Par ailleurs, certains soustypes ont été surabondamment employés, alors même que les spécimens archéologiques ne remplissaient pas l'ensemble des caractères stylistiques requis. Certes, en se basant sur les dessins, on opère un échantillonnage quantitatif pour les sites où la totalité des armatures n'est pas représentée. Une vérification a cependant montré que les proportions des types ne varient que très peu entre les dessins et les décomptes, ce qui a donc un faible impact sur la question des divisions stylistiques. Elle a également permis de parler de représentativité qualitative des sous-types puisqu'ils ont été dessinés dans leur ensemble. Cependant, il ne faut pas oublier qu'en fonction du mode 
de représentation graphique de l'armature, l'identification de sous-types précis peut poser problème.

La caractérisation d'entités culturelles s'est heurtée au caractère partial de la documentation à disposition. Souvent des « indices mésolithiques » sont signalés sans mentionner de cadre chronologique plus restreint et/ou sans description du mobilier - dont d'éventuelles armatures. Par conséquent, des sites du premier Mésolithique restent à mettre au jour par analyse de collections. L'ensemble de la documentation a permis de recenser 145 indices du premier Mésolithique, parmi lesquels 87 présentent tous les critères requis par cette étude - soit un corpus de 4312 armatures. Ces nombreux indices offrent de bonnes conditions pour tenter de définir des entités stylistiques en Centre-Ouest.

\section{Distribution spatiale des données}

La répartition des données recensées montre des zones d'occupation dense au sein de la vallée de la Charente, dans le bocage vendéen et le Sud-nantais, ainsi qu'au niveau de l'estuaire de la Loire (fig. 3). Ces concentrations ne reflètent toutefois que l'emplacement des investigations mentionnées dans la littérature au sens large. L'intense occupation de ces zones laisse présager un fort potentiel de sites à découvrir, ou à faire connaître, dans les départements de la Mayenne, de la Sarthe, de Maine-et-Loire et de la Charente, où les sites et armatures étudiables ne sont actuellement pas suffisamment nombreux pour en obtenir des informations concluantes. Pour les mêmes raisons, une caractérisation culturelle est exclue pour les départements des Deux-Sèvres et de la Vienne. La répartition des gisements obtenue est également influencée par des facteurs géologiques et topographiques - phénomènes d'érosion ou de recouvrement de sites, zones plus ou moins propices à la prospection. Les secteurs apparemment vides d'armatures peuvent aussi découler des techniques d'investigations employées, ou résulter de la spécialisation des activités des groupes mésolithiques. N'oublions pas pour finir que l'aire de répartition des données, et donc la définition des entités concernées, est limitée par l'immersion des bandes littorales de leurs territoires, suite à une hausse du niveau marin d'environ $60 \mathrm{~m}$ au cours du premier Mésolithique (Pirazzoli, 1991).

Afin de saisir les facteurs limitant la définition de territoires stylistiques, les gisements ont été classés en fonction de la quantité et de la qualité des informations qu’ils fournissaient. Près de $83 \%$ des indices proviennent de prospections, qui constituent la principale source de données concernant l'occupation du territoire au premier Mésolithique. Certaines de ces séries sont hétérogènes, regroupent peu de pièces et présentent le risque de sous-estimer les microlithes hyperminiaturisés. Il peut alors être difficile d'établir un dia- gnostic chronologique ou d'utiliser de manière pertinente les statistiques. Ce type d'opération étant insuffisant à lui seul, de futures fouilles, généralisant le tamisage systématique qui jusqu'alors a rarement été mis en œuvre, seraient grandement bénéfiques sur l'ensemble du Centre-Ouest pour mieux définir ses occupants au début de l'Holocène. En effet, si l'on s'en tient au système de hiérarchisation de G. Marchand ${ }^{4}$ (Marchand et al., 2004), seul la PierreSaint-Louis (Geay, Charente-Maritime) peut être considéré comme un site majeur servant de référence valable pour une bonne reconstitution paléo-ethnographique. Il ne faut cependant pas minimiser la valeur des informations livrées par des gisements tels que La Grange (Surgères, CharenteMaritime), Chez Jouneau (Brizambourg, Charente) (fig. 4), ou l'abri des Rocs (Bellefonds, Vienne).

\section{Caractérisation des territoires STYLISTIQUES DU PREMIER MÉsOlithiQUe en Poitou-Charentes et Pays-de-la-Loire}

Le premier Mésolithique tel qu'il est entendu ici couvre une période d'environ 2800 ans. Pour la cohérence de cette étude, il fallait donc tenter d'individualiser les séries d'un point de vue chronologique. La carence en références stratigraphiques fiables et le manque de datations par le radiocarbone dont souffre le Centre-Ouest n'ont pas simplifié cette tâche. Une certaine neutralité a été privilégiée dans la chronologie et les dénominations employées, ceci pour trois raisons :

- afin d'éviter d'attribuer à des industries des dates qui en réalité ne sont pas assurées, notamment à cause d'un effet plateau sur la courbe de calibration, ayant pour effet une concentration des dates vers $8800 \mathrm{BP}$;

- pour ne pas créer d'éventuelles scissions fictives à travers les appellations Mésolithique ancien et Mésolithique moyen (Valdeyron 1994);

- et parce que les travaux typologiques sur les grandes lignes évolutives des armatures sont basés sur des régions voisines (Rozoy, 1978a; Barbaza et al., 1991; Thévenin, 1991 ; Valdeyron, 1994).

La chronologie a donc été agencée en faciès dénués de bornes chronologiques fixes, bien que largement inspirés des variations typologiques proposées au sein de ces derniers travaux (fig. 5).

- Le faciès « $A$ » se définit essentiellement par un taux élevé de triangles isocèles, ou de morphologie proche, et par la présence marquée de pointes à base naturelle ou retouchée.

4. Il repose sur une tripartition : indices de sites/sites confirmés/sites majeurs : des indices ténus, on passe aux collections numériquement plus importantes puis à celles permettant l'élaboration d'un discours paléoethnographique. 


\begin{tabular}{|c|c|c|c|c|}
\hline No du site & $\left\{\begin{array}{c}\text { Noms du site et du } \\
\text { lieu dit }\end{array}\right.$ & Commune & Département & Faciès \\
\hline 1 & $\begin{array}{l}\text { Grotte de } \\
\text { Perrats }\end{array}$ & Agris & 16 & \\
\hline 2 & $\begin{array}{l}\text { Le Bois de } \\
\text { Douvesse }\end{array}$ & Bouteville & 16 & \\
\hline 3 & La Cassine & Edon & 16 & \\
\hline 4 & $\begin{array}{c}\text { Les } \\
\text { Renardières }\end{array}$ & Les Pins & 16 & \\
\hline 5 & Garde Epée & Saint-Brice & 16 & 1 \\
\hline 6 & Le Laquet & Aigrefeuille-d'Aunis & 17 & \\
\hline 7 & Archingeay & Archingeay & 17 & \\
\hline 8 & Chez Jouneau & Brizambourg & 17 & B \\
\hline 9 & Chez Gautret & Brizambourg & 17 & \\
\hline 10 & Les Prises & Brizambourg & 17 & B \\
\hline 11 & La Brousse & Brizambourg & 17 & \\
\hline 12 & Rochebrune & Brizambourg & 17 & I \\
\hline 13 & Chasserat & Cabariot & 17 & C \\
\hline 14 & \begin{tabular}{|c|}
$\begin{array}{c}\text { La Fontaine des } \\
\text { maçons }\end{array}$ \\
\end{tabular} & Champagne & 17 & \\
\hline 15 & \begin{tabular}{|c|}
$\begin{array}{c}\text { Au bois de } \\
\text { l'Eguille }\end{array}$ \\
\end{tabular} & Champagne & 17 & B \\
\hline 16 & L'Eguille & Champagne & 17 & B \\
\hline 17 & \begin{tabular}{|c|}
$\begin{array}{c}\text { La Pièce de la } \\
\text { Croix }\end{array}$ \\
\end{tabular} & Champagne & 17 & B \\
\hline 18 & Les Barrières & Champdolent & 17 & \\
\hline 19 & $\begin{array}{l}\text { Les Grandes } \\
\text { Prises }\end{array}$ & Corme-Royal & 17 & C \\
\hline 20 & $\begin{array}{l}\text { Le Bois } \\
\text { Barillet }\end{array}$ & Fenioux & 17 & B \\
\hline 21 & Les Périnets & Fenioux & 17 & \\
\hline 22 & $\begin{array}{l}\text { Le Grand } \\
\text { Plantis }\end{array}$ & La Fredière & 17 & \\
\hline 23 & \begin{tabular}{|c|}
$\begin{array}{c}\text { La Pierre Saint } \\
\text { Louis }\end{array}$ \\
\end{tabular} & Geay & 17 & B \\
\hline 24 & $\begin{array}{l}\text { Abri Piphrez, } \\
\text { Chez Guérin }\end{array}$ & Grandjean & 17 & A \\
\hline 25 & L'lle d'Erablais & Hiers-Brouage & 17 & I \\
\hline 26 & L'Antruère & Lussant & 17 & 1 \\
\hline 27 & $\begin{array}{c}\text { La Maison } \\
\text { Neuve }\end{array}$ & Lussant & 17 & C \\
\hline 28 & Guyarderie & Moragne & 17 & \\
\hline 29 & Les Charrauds & Les Nouillers & 17 & \\
\hline 30 & Les Ouchettes & Plassay & 17 & I \\
\hline 31 & Champon & $\begin{array}{c}\begin{array}{c}\text { Saint-Laurent-de-la- } \\
\text { Prée }\end{array} \\
\end{array}$ & 17 & B \\
\hline 32 & Champs Benon & Saint-Savinien & 17 & \\
\hline 33 & Chez Chenard & $\begin{array}{c}\text { Saint-Sulpice-en- } \\
\text { Cognac } \\
\end{array}$ & 17 & \\
\hline 34 & Peuillet & $\begin{array}{c}\text { Saint-Sulpice-en- } \\
\text { Cognac }\end{array}$ & 17 & C \\
\hline 35 & Sept Fonds & $\begin{array}{c}\text { Saint-Sulpice-en- } \\
\text { Cognac }\end{array}$ & 17 & C \\
\hline 36 & Le Prieuré & La Borderie & 17 & \\
\hline 37 & Charcognier & Surgères & 17 & l \\
\hline 38 & La Grange & Surgères & 17 & C \\
\hline
\end{tabular}

\begin{tabular}{|c|c|c|c|c|}
\hline 39 & $\begin{array}{l}\text { Les Fontaines } \\
\text { Pissoche }\end{array}$ & Taillant & 17 & I \\
\hline 40 & Puyssoteau & Tonnay-Charente & 17 & B \\
\hline 41 & Le Cloux & Tonnay-Charente & 17 & C \\
\hline 42 & $\begin{array}{l}\text { La Grande } \\
\text { Chauvinière }\end{array}$ & Tonnay-Charente & 17 & \\
\hline 43 & $\begin{array}{l}\text { La Grande } \\
\text { Pièce }\end{array}$ & La Vergne & 17 & \\
\hline 44 & Bel Air & Villars-les-Bois & 17 & 1 \\
\hline 45 & Chez Bonpain & Villars-les-Bois & 17 & \\
\hline 46 & Fontbelle & Villars-les-Bois & 17 & $\mathrm{~B}$ \\
\hline 47 & Peu Deis & Villars-les-Bois & 17 & \\
\hline 48 & La Meule (2) & Arthon-en-Retz & 44 & 1 \\
\hline 49 & La Gatine & Corcoué-sur-Longe & 44 & \\
\hline 50 & $\begin{array}{l}\text { Le Fief des } \\
\text { Rivières (2) }\end{array}$ & Corcoué-sur-Longe & 44 & C \\
\hline 51 & $\begin{array}{l}\text { Le Fief de la } \\
\text { Grange }\end{array}$ & Corcoué-sur-Longe & 44 & \\
\hline 52 & La Gautrais & Crossac & 44 & \\
\hline 53 & Les Garennes & Geneston & 44 & B \\
\hline 54 & Les Crolières & Guérande & 44 & B \\
\hline 55 & Clis A & Guérande & 44 & 1 \\
\hline 56 & Clis B & Guérande & 44 & I \\
\hline 57 & Clis C & Guérande & 44 & 1 \\
\hline 58 & Gras & Guérande & 44 & B \\
\hline 59 & Sandun & Guérande & 44 & c \\
\hline 60 & Les Cléons & Haute-Goulaine & 44 & 1 \\
\hline 61 & $\begin{array}{l}\text { Près Le } \\
\text { passage à } \\
\text { niveau }\end{array}$ & Machecoul & 44 & \\
\hline 62 & La Caillette & Machecoul & 44 & B \\
\hline 63 & $\begin{array}{l}\text { Les Etangs de } \\
\text { la Brenière }\end{array}$ & Montbert & 44 & A \\
\hline 64 & La Majoire A & Montbert & 44 & B \\
\hline 65 & La Majoire B & Montbert & 44 & 1 \\
\hline 66 & La Majoire C & Montbert & 44 & B \\
\hline 67 & La Majoire D & Montbert & 44 & $\mathrm{~B}$ \\
\hline 68 & La Majoire E & Montbert & 44 & I \\
\hline 69 & Port Nabé & Piriac & 44 & I \\
\hline 70 & La Petite lle & Pontchâteau & 44 & A \\
\hline 71 & Le Châtelet & Préfailles & 44 & I \\
\hline 72 & L'Anse du Sud & Préfailles & 44 & I \\
\hline 73 & Préfailles (2) & Préfailles & 44 & 1 \\
\hline 74 & My & Saint-Anne-sur-Brivet & 44 & \\
\hline 75 & Kerlo & Saint-Lyphard & 44 & $\mathrm{~B}$ \\
\hline 76 & $\begin{array}{l}\text { La Butte aux } \\
\text { Pierres }\end{array}$ & Saint-Joachim & 44 & I \\
\hline 77 & $\begin{array}{l}\text { L'lle de la } \\
\text { Perrière }\end{array}$ & Saint-Nazaire & 44 & c \\
\hline
\end{tabular}




\begin{tabular}{|c|c|c|c|c|}
\hline 78 & $\begin{array}{c}\text { Caïrn de } \\
\text { Dissignac }\end{array}$ & Saint Nazaire & 44 & C \\
\hline 79 & $\begin{array}{c}\text { Les Vingt-deux } \\
\text { Boisselées }\end{array}$ & Saint-Père-en-Retz & 44 & A \\
\hline 80 & La Fillauderie & Saint-Père-en-Retz & 44 & B \\
\hline 81 & L'Organais & $\begin{array}{l}\text { Sainte-Reine-de- } \\
\text { Bretagne }\end{array}$ & 44 & A \\
\hline 82 & La Garne & Vieillevigne & 44 & B \\
\hline 83 & Les Chaloignes & Mozé-sur-Louet & 49 & \\
\hline 84 & La Pommeraye & La Pommeraye & 49 & I \\
\hline 85 & La Miquenaie & $\begin{array}{l}\text { Saint Michel-et- } \\
\text { Chanveaux }\end{array}$ & 49 & l \\
\hline 86 & Les Pâtures & Villevêque & 49 & C \\
\hline 87 & $\begin{array}{l}\text { Le Point du } \\
\text { jour }\end{array}$ & Duneau & 72 & I \\
\hline 88 & Les Fraisiers & Dureil & 72 & I \\
\hline 89 & La Croix Verte & Montmirail & 72 & B \\
\hline 90 & $\begin{array}{l}\text { La Roche } \\
\text { Châtain }\end{array}$ & Chantecorps & 79 & \\
\hline 91 & L'Aurière & Ménigoute & 79 & \\
\hline 92 & $\begin{array}{c}\text { Le Bois } \\
\text { Pouvreau }\end{array}$ & Ménigoute & 79 & \\
\hline 93 & La Boutinière & Ménigoute & 79 & \\
\hline 94 & La Brouinière & Ménigoute & 79 & \\
\hline 95 & Puy Albert & Moulins & 79 & l \\
\hline 96 & $\begin{array}{c}\text { Le Champ de } \\
\text { la Vigne }\end{array}$ & $\begin{array}{l}\text { Saint André-sur- } \\
\text { Sèvre } \\
\end{array}$ & 79 & B \\
\hline 97 & $\begin{array}{c}\text { La Guilbaudière } \\
\text { (1) }\end{array}$ & $\begin{array}{l}\text { Saint-Martin-du- } \\
\text { Fouilloux }\end{array}$ & 79 & \\
\hline 98 & $\begin{array}{c}\text { La Guilbaudière } \\
\text { (2) }\end{array}$ & $\begin{array}{l}\text { Saint-Martin-du- } \\
\text { Fouilloux }\end{array}$ & 79 & \\
\hline 99 & $\begin{array}{c}\text { La Guilbaudière } \\
\text { (3) }\end{array}$ & $\begin{array}{l}\text { Saint-Martin-du- } \\
\text { Fouilloux }\end{array}$ & 79 & \\
\hline 100 & $\begin{array}{c}\text { La Haute } \\
\text { Jarrière }\end{array}$ & $\begin{array}{l}\text { Saint-Martin-du- } \\
\text { Fouilloux } \\
\end{array}$ & 79 & \\
\hline 101 & $\begin{array}{l}\text { Le Bois Tollu } \\
\text { (1) }\end{array}$ & Vasles & 79 & \\
\hline 102 & $\begin{array}{l}\text { Le Bois Tollu } \\
\text { (2) }\end{array}$ & Vasles & 79 & \\
\hline 103 & $\begin{array}{c}\text { Les } \\
\text { Bourdinières }\end{array}$ & Vasles & 79 & \\
\hline 104 & $\begin{array}{l}\text { Les Fougères } \\
\text { (1) }\end{array}$ & Vasles & 79 & \\
\hline 105 & Les Fradinières & Vasles & 79 & \\
\hline 106 & $\begin{array}{c}\text { La Petite } \\
\text { Poitevinière (3) }\end{array}$ & Vasles & 79 & B \\
\hline 107 & La Prouterie & Vasles & 79 & \\
\hline 108 & Vaulifier & Vasles & 79 & \\
\hline 109 & Gauziot & Vausseroux & 79 & \\
\hline 110 & Malépine & Vautebis & 79 & \\
\hline 111 & $\begin{array}{c}\text { Entre } \\
\text { Boislivière et } \\
\text { les Brandes }\end{array}$ & L'Aiguillon-sur-Vie & 85 & B \\
\hline
\end{tabular}

\begin{tabular}{|c|c|c|c|c|}
\hline 112 & Les Emerillères & Beaufou & 85 & B \\
\hline 113 & Le Grand Pâtis & Boulogne & 85 & I \\
\hline 114 & $\begin{array}{c}\text { Maison à } \\
\text { gauche }\end{array}$ & Boulogne & 85 & I \\
\hline 115 & Maison à droite & Boulogne & 85 & 1 \\
\hline 116 & Les Vergères & Boulogne & 85 & B \\
\hline 117 & Plage de Bâtard & Brétignolles & 85 & \\
\hline 118 & La Maucarière & Chambretaud & 85 & \\
\hline 119 & $\begin{array}{l}\text { Saint Jean } \\
\text { d'Orbestier }\end{array}$ & Château-d'Olonne & 85 & I \\
\hline 120 & Bel-Air & Château-d'Olonne & 85 & 1 \\
\hline 121 & Guillonnière & Dompierre & 85 & 1 \\
\hline 122 & $\begin{array}{l}\text { Chatelliers- } \\
\text { Châteaumur }\end{array}$ & $\begin{array}{l}\text { Chatelliers- } \\
\text { Châteaumur }\end{array}$ & 85 & \\
\hline 123 & La Ganache & Les Epesses & 85 & I \\
\hline 124 & $\begin{array}{c}\text { Le Moulin de la } \\
\text { Grange }\end{array}$ & Les Epesses & 85 & 1 \\
\hline 125 & La Soudelache & La Gaubretière & 85 & B \\
\hline 126 & La Pierre Levée & Nieul-sur-L'Autize & 85 & 1 \\
\hline 127 & Luzeronde & Noimoitier-en-l'Ile & 85 & \\
\hline 128 & Le Moulin Neuf & Roche-sur-Yon & 85 & B \\
\hline 129 & L'Ouvrardière & $\begin{array}{l}\text { Saint Denis-la- } \\
\text { Chevasse }\end{array}$ & 85 & I \\
\hline 130 & $\begin{array}{l}\text { Le Bois des } \\
\text { Jarries }\end{array}$ & $\begin{array}{l}\text { Saint-Mars-la- } \\
\text { Réorthe }\end{array}$ & 85 & B \\
\hline 131 & La Glanière & $\begin{array}{l}\text { Saint-Mesmin-le- } \\
\text { Vieux }\end{array}$ & 85 & B \\
\hline 132 & Bourgenay & Talmont-Saint-Hilaire & 85 & \\
\hline 133 & Sangle (2) & La Verrie & 85 & c \\
\hline 134 & Les Coux & La Flocellière & 85 & \\
\hline 135 & Le Tillay Mandin & Mallièvre & 85 & \\
\hline 136 & L'Abri des Rocs & Bellefonds & 86 & l \\
\hline 137 & Gioux & Chauvigny & 86 & I \\
\hline 138 & Le Grand Pré & Curzay-sur-Vonne & 86 & \\
\hline 139 & L'Essart & Poitiers & 86 & \\
\hline 140 & $\begin{array}{l}\text { La Grande } \\
\text { Roche de la } \\
\text { Plématrie }\end{array}$ & Quincay & 86 & C \\
\hline 141 & Marconnay & Sanxay & 86 & \\
\hline 142 & $\begin{array}{l}\text { La Grotte } \\
\text { Chaffaud }\end{array}$ & Savigné & 86 & 1 \\
\hline 143 & Le Parc & Scorbe-Clairvaux & 86 & \\
\hline 144 & $\begin{array}{c}\text { La Garenne, abri } \\
1\end{array}$ & Valdivienne & 86 & \\
\hline 145 & Le Peu & Vaux-sur-Vienne & 86 & \\
\hline
\end{tabular}

Tableau 2 : Liste des sites considérés. Les gisements étudiés ici sont mentionnés en gras et leur faciès est précisé. Tableau 2: List of sites studied. Those considered here are in bold and their facies is specified. 


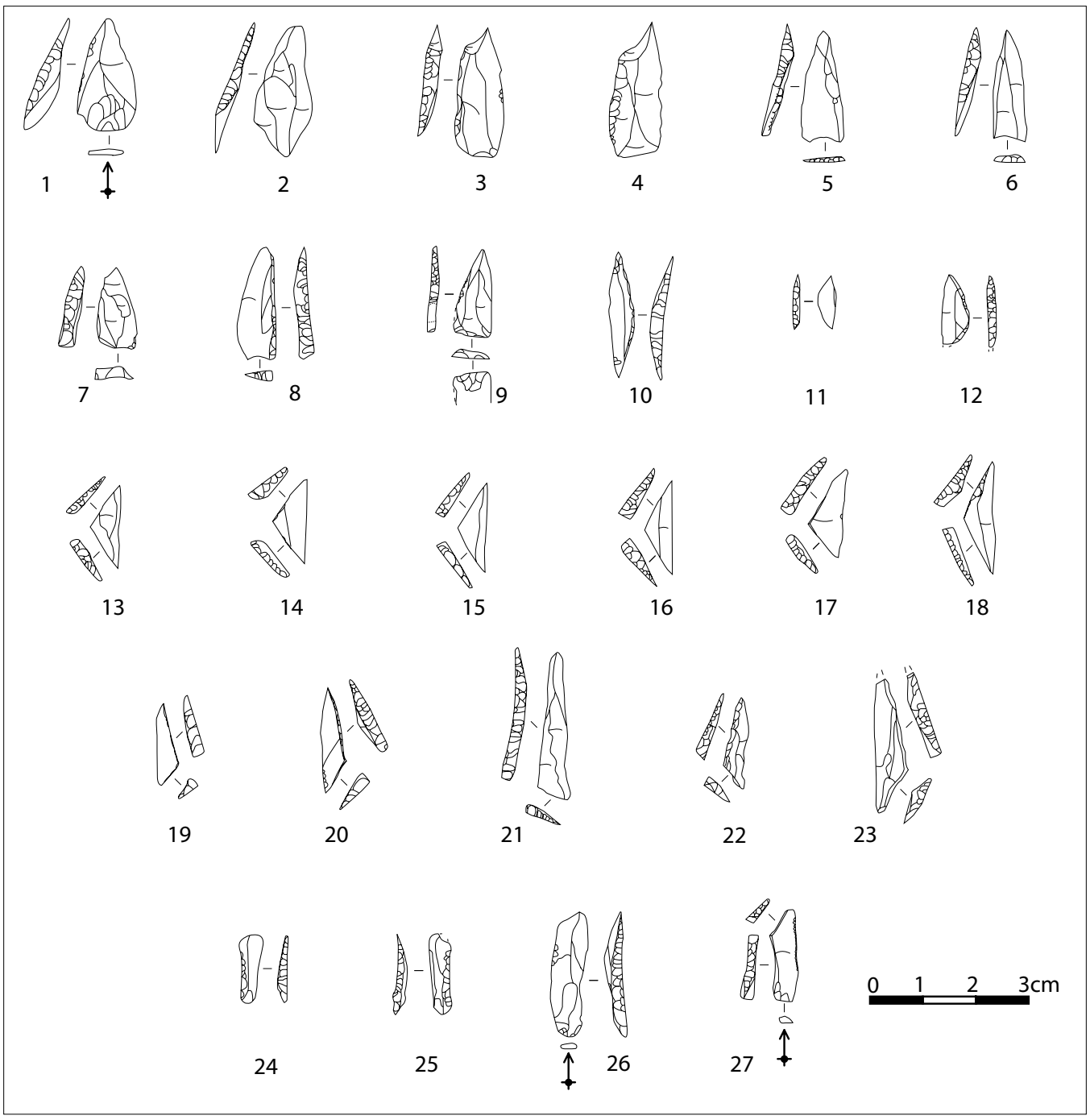

Figure $4:$ Armatures. 1, 2 : pointes à base naturelle; 3, $4:$ pointes de Chaville; 5-9: pointes à base retouchée ; 10 : pointe fusiforme ; 11, 12 : segments de cercle; 13-18: triangles isocèles; 19-23: triangles scalènes ; 24-26 : lamelles à bord abattu; 27 : lamelle-scalène.

Provenances : 1-3, 5, 6, 11, 13-17, 19-21, 24, 27 : Chez Jouneau (Brizambourg) ; 4, 9, 10, 22, 23 : SeptFonds (Saint-Sulpice-en-Cognac) ; 7, 18, 25 : Fontbelle (Villars-les-Bois) ; 8, 12, 26 : Peuillet (Saint-Sulpiceen-Cognac).

Figure 4: Arrowheads. 1, 2: points with natural base; 3, 4: Chaville points; 5-9: points with retouched base; 10: fusiform point; 11, 12: segments; 13-18: isoscele triangles; 19-23: scalene triangles; 24-26: backed bladelets; 27: scalene-blade.

- Le faciès « $\mathrm{B}$ » s'en distingue principalement par ses triangles qui suivent plus volontiers un modèle scalène diversifié.

- Au faciès " $\mathrm{C}$ ", les triangles isocèles sont absents alors que les scalènes sont effilés et miniaturisés. Lorsque des pointes apparaissent dans les collections, celles à base naturelle sont minoritaires par rapport aux modèles à base retouchée.

Le mélange de composantes culturelles dans plus de deux tiers des cas n'a pas facilité l'attribution à un faciès; ce n'est que lorsqu'une composante majoritaire émergeait que ces collections y ont été rattachées (table. 2).

\section{Introduction au faciès « $A$ »}

Environ $7 \%$ seulement des gisements de notre corpus intègrent avec certitude ce faciès. Les industries concernées se concentrent essentiellement en Loire-Atlantique, où les triangles isocèles représentent de la moitié à plus des trois quarts $\mathrm{du}$ fonds microlithique. Le site de la Petite-Île (Pontchâteau, 


\begin{tabular}{|c|c|c|c|c|c|c|}
\hline Types & & 夏 & 睋 & & 直 & $\begin{array}{l}\text { Gisements de référence } \\
\text { en Centre-Ouest }\end{array}$ \\
\hline A & $X$ & $x$ & & $X$ & $\mathrm{x}$ & $\begin{array}{l}\text { Les Vingt-Deux Boisselées, Saint-Père-en-Retz } \\
\text { L'Organais, Sainte-Reine-de-Bretagne }\end{array}$ \\
\hline B & $x$ & $X$ & $X$ & $X$ & $X$ & $\begin{array}{c}\text { La Pierre-Saint-Louis, Geay Les Majoires, Montbert } \\
\text { Chez Jouneau, Brizambourg }\end{array}$ \\
\hline C & & $x$ & $X$ & $x$ & $X$ & La Grange, Surgères \\
\hline
\end{tabular}

Figure 5 : Principaux types d'armatures des faciès " $\mathrm{A}$ », « $\mathrm{B}$ » et « $\mathrm{C}$ ».

Figure 5: Main arrowheads types of facieses " $A$ ", " $B$ " and " $C$ ".

Loire-Atlantique), qui pourrait en faire partie même si une certaine prudence s'impose à cause d'un mobilier numériquement restreint, est le seul à accueillir des triangles équilatéraux (fig. 6, $\mathrm{n}^{\circ}$ 19, 20). La structure industrielle des gisements de Loire-Atlantique, à l'image de celle des Étangs de la Brenière (Montbert), ne s'oppose pas franchement à celle des sites méridionaux de Jacques (Naujan-et-Postiac, Gironde) et de Fontfaurès (Lentillac-Lauzac, Lot) - couche 6b, ce qui n'empêche pas certaines divergences entre les armatures sauveterriennes et ligériennes (Valdeyron, 1994). On peut ainsi évoquer pour les sites de Loire-Atlantique, dont les VingtDeux Boisselées (Saint-Père-en-Retz) ou L'Organais (SainteReine-de-Bretagne), la grande discrétion des lamelles à bord(s) abattu(s). Les pointes à base retouchée, quant à elles, y sont en moyenne six fois plus représentées que dans la couche $6 \mathrm{~b}$ de Fontfaurès. Pareillement, les pointes à base naturelle sont d'un tiers moins fréquentes dans les sites méridionaux. La nature exacte des liens entre le Sauveterrien et les entités techniques ligériennes reste donc à éclaircir.

La panoplie microlithique des groupes humains du sud du Centre-Ouest est moins bien appréhendée. Les informations obtenues à partir de gisements comme l'abri Piphrez (Grandjean, Charente-Maritime) sont à traiter avec prudence : le fait que le pourcentage des triangles isocèles et scalènes y soit équilibré mais que l'on soit également en présence d'un bon nombre de microlithes très effilés évoque un mélange entre différentes composantes chronologiques récoltées après destruction de l'abri. L'importance des lamelles à bord(s) abattu(s) rappelle la sphère méridionale.

\section{Distinction d'entités stylistiques au sein du faciès « $B$ »?}

L'importance des données récoltées permet une bonne caractérisation stylistique pour ce faciès. Elle n'est cependant envisageable que pour la Loire-Atlantique, la Vendée et les Charentes ${ }^{5}$, zones qui se démarquent de manière plus ou moins sensible les unes des autres ${ }^{6}$. Parmi les caractéristiques qui unissent cependant les corpus de ces départements, on constate la faible part accordée aux segments de cercle (3,3\%, tabl. 3).

Les pointes de Sauveterre (fig. 7; tabl. 4) apparaissent dans très peu de gisements et forment à chaque fois un groupe trop limité pour être significatif. L'exemplaire du site de la Majoire C (Montbert, Loire-Atlantique) (fig. 6, $\mathrm{n}^{\circ}$ 51) demeure un indice isolé au nord des Charentes et l'absence de sa partie basse interdit un rattachement strict à ce sous-type.

Les pointes à base retouchée quant à elles sont localisées dans l'ensemble de ces départements et dans des proportions comparables, montrant le statut marqué de ce type. Dans de nombreuses publications, elles reçoivent la qualification de tardenoisiennes alors qu'en réalité on observe majoritairement une morphologie inverse - à savoir une partie basse bombée, les bords au niveau de l'extrémité apicale étant plus rectilignes.

Au niveau des triangles scalènes, l'importance des spécimens à petite troncature concave s'illustre par leur présence dans deux tiers des sites recensés et une proportion d'un cinquième au sein du type. La quasi-absence des triangles de Montclus - scalènes effilés au troisième côté retouché apparus dès le "Sauveterrien ancien évolué » en faible proportion (Valdeyron, 1994) - est également un des traits caractéristiques du Centre-Ouest : pour le faciès « B », on n'en dénombre que deux spécimens, deux autres étant des armatures apparentées (fig. 8).

5. L'étude a regroupé l'ensemble des séries des départements de la Charente et de la Charente-Maritime compte tenu d'une part de la proximité géographique des indices et gisements du premier Mésolithique, d'autre part de la ressemblance typologique des armatures.

6. La description des collections par départements ne se base pas sur le découpage administratif mais sur l'observation de distinctions typologiques. 


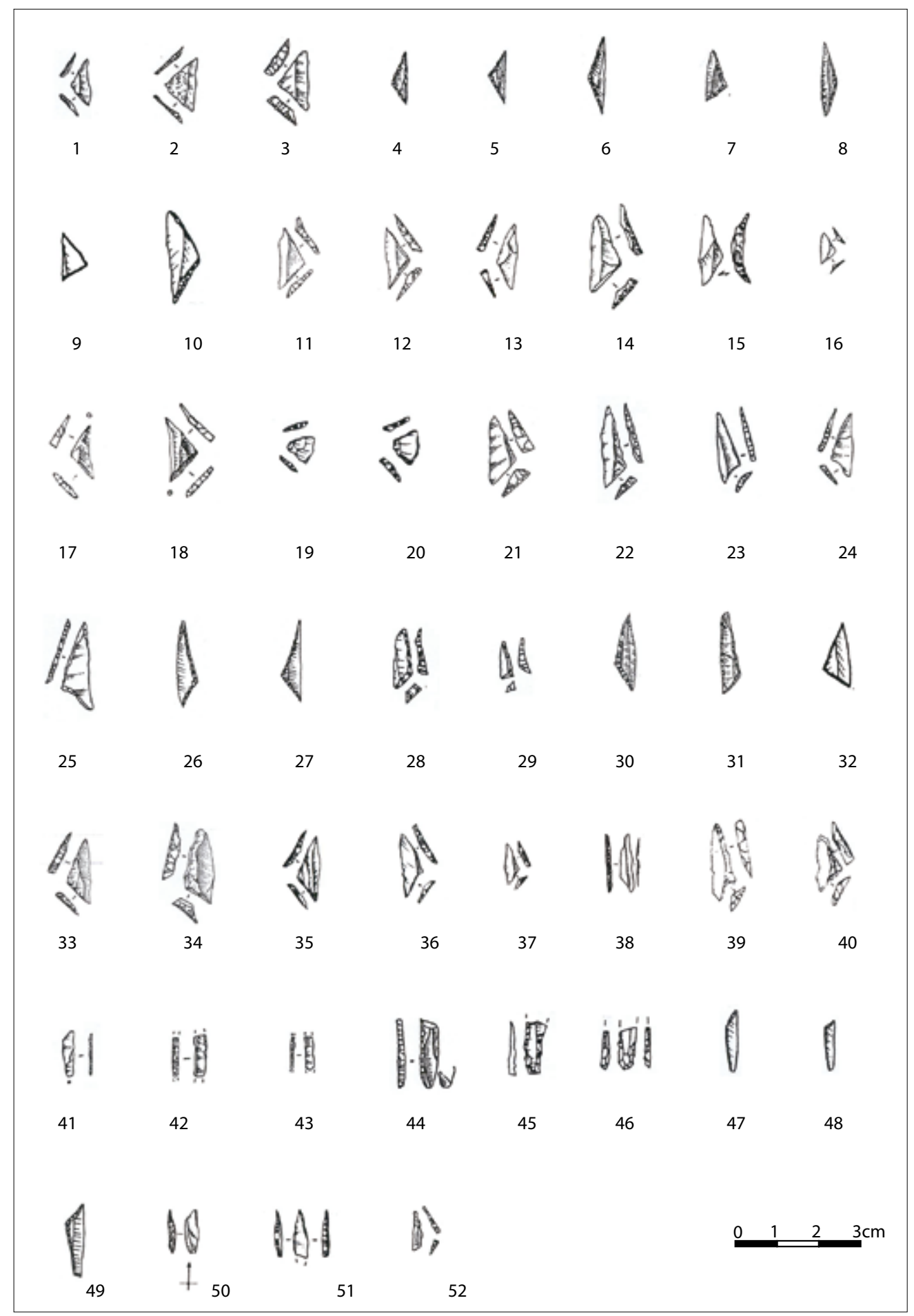

Figure 6 : Armatures. 1-18: triangles isocèles; 19, 20 : triangles équilatéraux; 21-40: triangles scalènes; 41-46: lamelles à bord abattu; 47-50 : lamelles-scalènes; 51 : pseudo-pointe de Sauveterre; 52 : triangle de Montclus.

Provenances : 1-3, 21-25, 41-44 : La Pierre-Saint-Louis (Geay); 4-6, 26, 27, 47-49 : abri Piphrez (Grandjean); 7, 8, 30, 31 : abri des Rocs (Bellefonds) ; 9, 10, 32 : Les Crolières (Guérande); 11, 12 : étang de la Brenière (Montbert); 13, 14, 51 : La Majoire C (Montbert); 15 : Les Vingt-deux Boisselées (Saint-Père-en-Retz); 16, 37, 38, 50 : La Garne (Vieillevigne); 17, 18 : L'Organais (Sainte-Reine-de-Bretagne); 19, 20 : La Petite-Île (Pontchâteau) ; 28, 29, 45, 46 : La Grange (Surgères); 33, 34 : La Majoire A (Montbert); 35, 36 : La Majoire D (Montbert); 39 , 40 : Les Vergères (Boulogne); 52 : Le Bois des Jarries (Saint-Mars-la-Réorthe) (dessins issus des rapports et publications originels)

Figure 6: Arrowheads. 1-18: isosceles triangles; 19, 20: equilateral triangles; 21-40: scalene triangles; 41-46: backed bladelets; 47-50: scaleneblades ; 51: pseudo Sauveterrian point; 52: Montclus triangle (drawings from original publications and reports). 


\begin{tabular}{|c|c|c|c|c|c|}
\hline & Loire-Atlantique & $\begin{array}{c}\text { Loire-Atlantique, } \\
\text { Nord-Loire }\end{array}$ & $\begin{array}{c}\text { Loire-Atlantique, } \\
\text { Sud-Loire }\end{array}$ & Vendée & Charentes \\
\hline Pointe à base naturelle & 34,1 & 45,1 & 27,8 & 23,3 & 8,8 \\
\hline Pointe à base retouchée & 17,3 & 17,7 & 17,1 & 21,4 & 12,5 \\
\hline Pointe fusiforme & 2,7 & 3,3 & 2,4 & 1,3 & 0,2 \\
\hline Segment de cercle & 4,7 & 4,2 & 5,0 & 3,7 & 2,5 \\
\hline Lamelle à bord(s) abattu(s) & 3,1 & 0,4 & 4,7 & 2,4 & 8,3 \\
\hline Triangle scalène & 26,0 & 20,5 & 29,1 & 25,4 & 49,9 \\
\hline Triangle isocèle & 12,1 & 8,8 & 13,9 & 22,5 & 17,8 \\
\hline Total & 100,0 & 100,0 & 100,0 & 100,0 & 100,0 \\
\hline
\end{tabular}

Tableau 3 : Proportion (en pourcentages) d'armatures dans les collections du premier Mésolithique en LoireAtlantique (globalement, nord-Loire et sud-Loire), Vendée, et Charentes pour les faciès " $\mathrm{A}$ » et « B ». Table 3: Percentages of arrowheads in the first Mesolithic series for Loire-Atlantique (in general, north and south of the Loire), Vendée and the Charentes for facieses " $A$ " and " $B$ "

\begin{tabular}{|c|c|c|c|c|c|c|}
\hline & Loire-Atlantique & $\begin{array}{c}\text { Loire-Atlantique, } \\
\text { Nord-Loire }\end{array}$ & $\begin{array}{c}\text { Loire-Atlantique, } \\
\text { Sud-Loire }\end{array}$ & Vendée & Charentes & Total \\
\hline Pointe de Chaville & 48,9 & 10,2 & 38,7 & 14,7 & 36,4 & 100 \\
\hline Pointe de la Majoire & 74,6 & 1,5 & 73,1 & 16,4 & 9 & 100 \\
\hline Pointe de Sauveterre & 0 & 0 & 0 & 14,3 & 85,7 & 100 \\
\hline
\end{tabular}

Tableau 4 : Proportions (en pourcentages) des pointes de Chaville, de la Majoire et de Sauveterre en Loire-Atlantique (globalement, nord-Loire et sud-Loire), Vendée et Charentes pour les faciès « $\mathrm{A}$ » et « $\mathrm{B}$ ».

Table 4: Percentages of Chaville, Majoire and Sauveterre points for Loire-Atlantique (general, north and south of the Loire), Vendée and the Charentes for facieses " $A$ " and " $B$ ".

\section{Le fonds microlithique des Charentes}

Il s'individualise par une large majorité de triangles - avec une préférence marquée pour les modèles scalènes - et un faible nombre voire une quasi-absence des pointes d'allure fusiforme ou à base naturelle (tabl. 3). De plus, $81,1 \%$ des lamelles à bord(s) abattu(s) du corpus total sont issues de cette zone. Une trentaine d'armatures ont une morphologie qui oscille entre les triangles scalènes et les lamelles à bord(s) abattu(s) tronquées ${ }^{7}$. Ces lamelles-scalènes se concentrent exclusivement dans les Charentes à l'exception d'une découverte à La Garne (Vieillevigne, Loire-Atlantique : fig. 6, $n^{\circ} 50$ ). Enfin, les pointes à base façonnée par retouches inverses se retrouvent dans presque tous les gisements charentais; leur proportion au sein des pointes à base retouchée $(30,8 \%)$ y est quatre à douze fois plus importante qu'en Pays-de-la-Loire.

7. Ces lamelles-scalènes se distinguent des triangles scalènes par l'absence de réunion du bord abattu et du côté opposé à la troncature oblique. Leur troncature n'est pas transversale mais bien oblique contrairement aux lamelles à bord(s) abattu(s) tronquées.

\section{Les armatures de Loire-Atlantique}

Ce sont les pointes à base naturelle qui prédominent ici. Près de la moitié des pointes de Chaville - à piquant-trièdre brut ou peu rectifié - du Centre-Ouest a été trouvée en LoireAtlantique et plus particulièrement au sud du fleuve (tabl. 4; fig. 9). Au sud de La Roche-sur-Yon, elles n'apparaissent que dans de rares sites, à savoir La Pierre-Saint-Louis et ChezJouneau; en revanche elles y sont assez nombreuses.

La conservation du piquant-trièdre brut sur les pointes à base retouchée est également plus marquée en Loire-Atlantique $\left(18,4 \%\right.$ de ces pointes $\left.{ }^{8}\right)$, ce caractère apparaissant quatre fois moins sur les armatures charentaises. Le même type de schéma s'applique aux pointes à base décalée dites de Vergères (Dugast et al., 2000). Leur morphologie reste très variable sur l'ensemble de la zone étudiée; d'éventuelles précisions dans la définition de ce sous-type, en lui associant d'autres critères, pourraient permettre de mieux évaluer les interactions entre le site éponyme (Boulogne, Vendée), où elles sont nombreuses (fig. 11, $\mathrm{n}^{\circ} 46,47$ ), et les autres gisements qui en accueillent une proportion plus faible. Pour ce qui concerne la conca-

8. Ceci n'est que le pourcentage minimum de conservation des piquantstrièdres, à cause des cassures au niveau des extrémités apicales. 


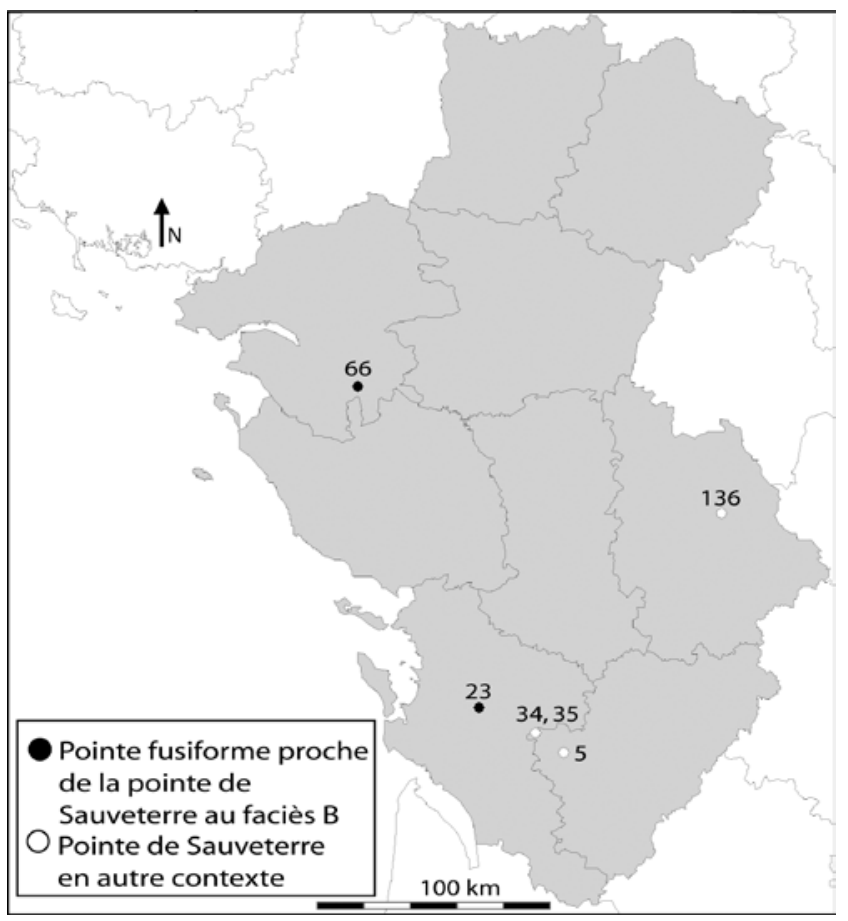

Figure 7 : Répartition spatiale des pointes de Sauveterre. Figure 7: Spatial distribution of Sauveterrian points.

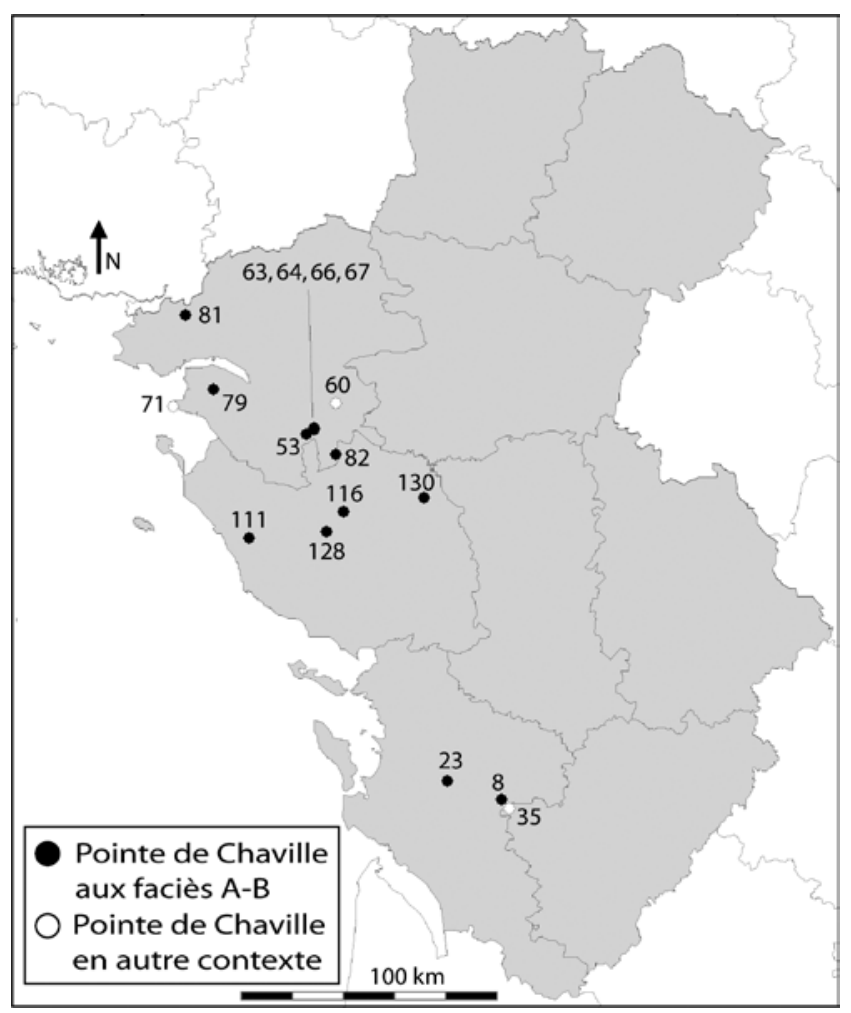

Figure 9 : Répartition spatiale des pointes de Chaville. Figure 9: Spatial distribution of Chaville points.

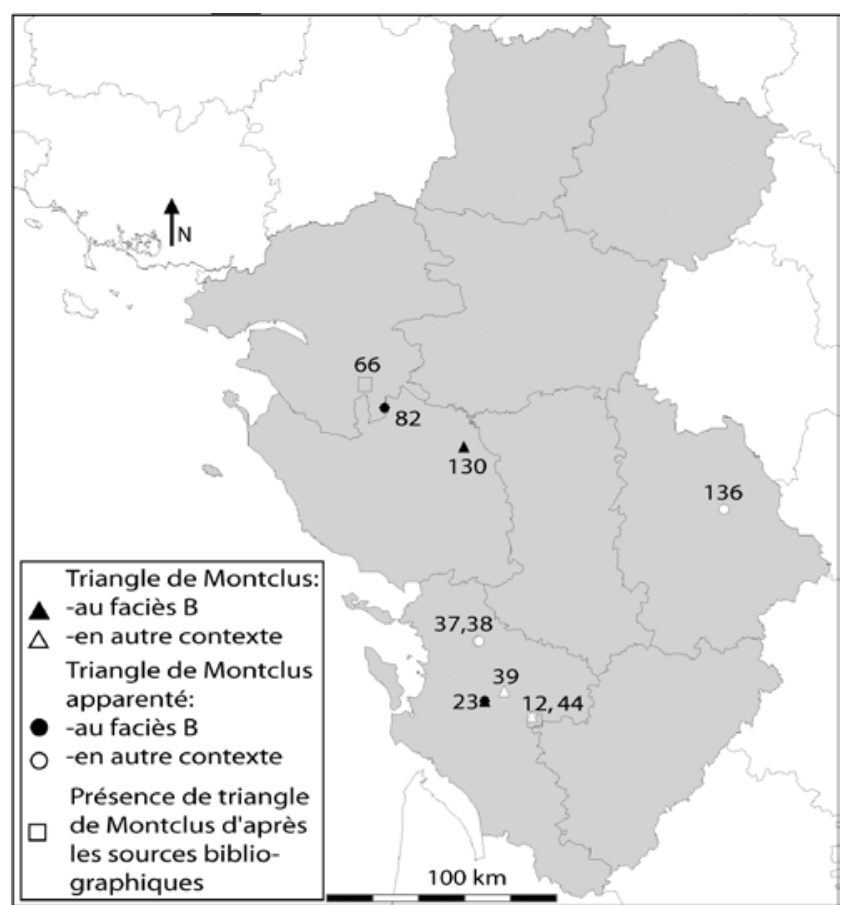

Figure 8 : Répartition spatiale des triangles de Montclus. Figure 8: Spatial distribution of Montclus triangles.

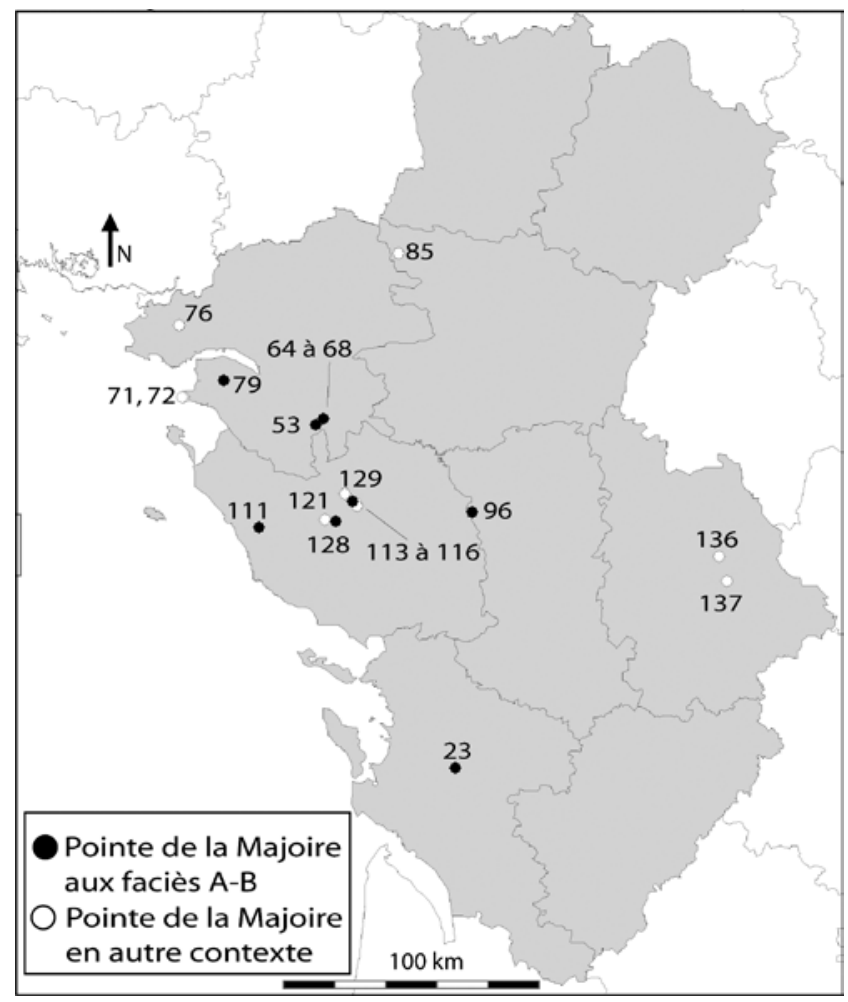

Figure 10 : Répartition spatiale des pointes de la Majoire. Figure 10: Spatial distribution of Majoire points. 


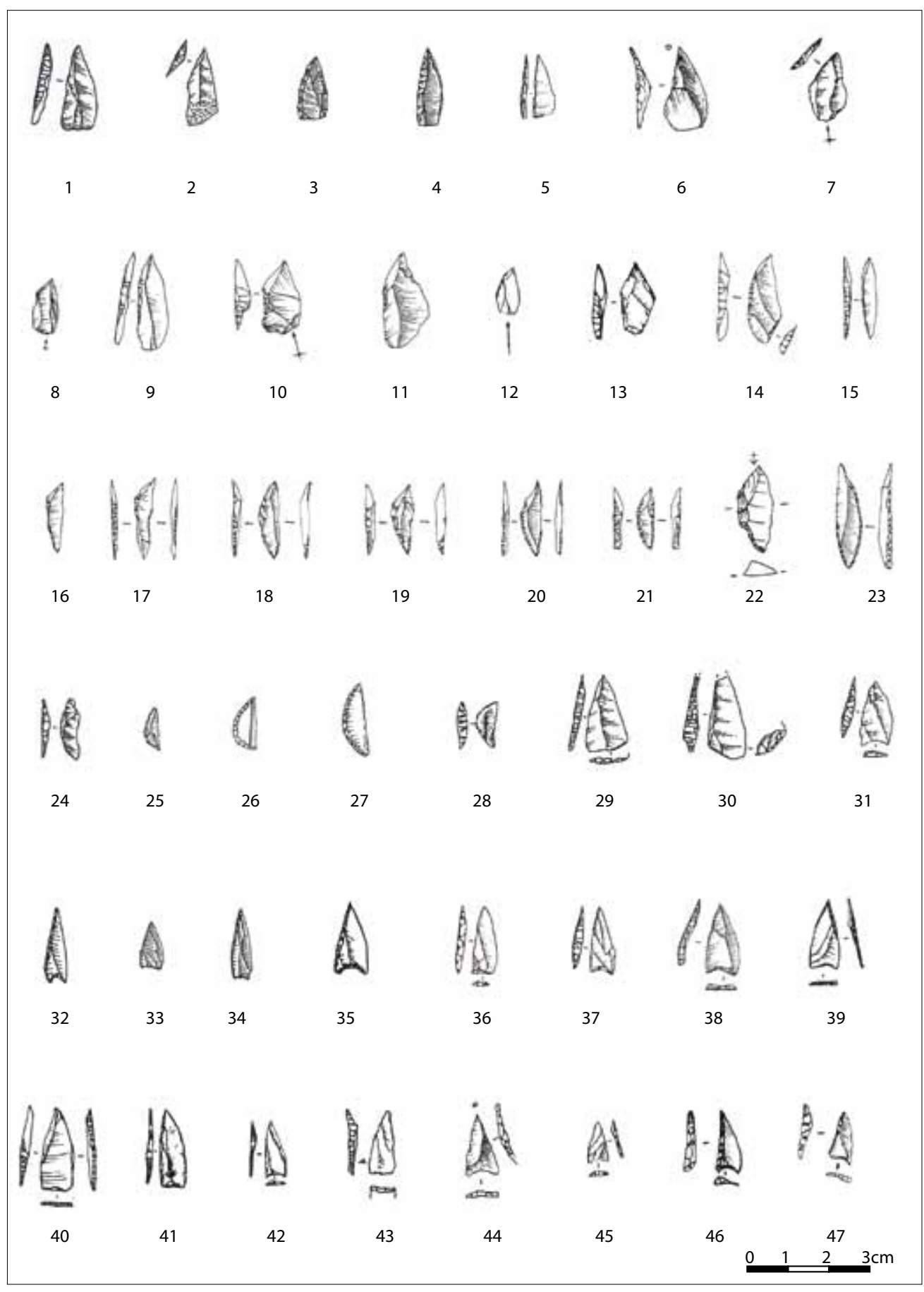

Figure 11 : Armatures. 1-7: pointes à base naturelle ; 8-13: pointes de Chaville; 14-23: pointes de la Majoire ; $24-28:$ segments de cercle; 29-47 : pointes à base retouchée.

Provenances : 1, 2, 14, 15, 24, 29-31 : La Pierre-Saint-Louis (Geay) ; 3, 4, 16, 25, 33, 34 : L'Abri des Rocs (Bellefonds); 5, 9, 36, 37 : Les Étang de la Brenière (Montbert) ; 6, 44 : L'Organais (Sainte-Reine-de-Bretagne); 7, 13, 46, 47 : Les Vergères (Boulogne) ; 8 : Les Garennes (Geneston); 10, 11, 38 : La Majoire A (Montbert); 12, $45:$ La Garne (Vieillevigne); 17 : Gioux (Chauvigny); 18, 19: La Majoire B (Montbert); 20, 21, 39, 40 : La Majoire C (Montbert); 22 : La Butte-aux-Pierres (Saint-Joachim-en-Brière); 23 : La Miquenaie (SaintMichel-et-Chanveaux); 26, 27 : Le Châtelet (Préfailles); 28 : Le Bois des Jarries (Saint-Mars-la-Réorthe); 32 : l'Abri Piphrez (Grandjean); 35 : Les Crolières (Guérande) ; 41, 42 : La Majoire D (Montbert) ; 43 : Les Vingt-deux Boisselées (Saint-Père-en-Retz) (dessins issus des rapports et publications originels).

Figure 11: Arrowheads. 1-7: points with natural base; 8-13: Chaville points; 14-23: Majoire points; 24-28: segments; 29-47: points with retouched base (drawings from original publications and reports). 
vité de la base, une distinction nette s'affirme : très en vogue en Loire-Atlantique et en Vendée $(60,7 \%)$, ce caractère est beaucoup moins apprécié en Charentes (18,8\%). Ce facteur, déjà évoqué auparavant (Bazin et al., 1995; Gouraud, 1996; Marchand et al., 1998), semble permettre une différenciation entre les entités stylistiques septentrionales et méridionales du Centre-Ouest.

Les pointes fusiformes constituent quant à elles une faible part du corpus de Loire-Atlantique. Toutefois, il est intéressant de noter qu'à l'échelle du Centre-Ouest elles ne représentent pas moins des deux tiers de ce type. Parmi les pointes fusiformes, celles de la Majoire, au piquanttrièdre brut ou peu régularisé, se concentrent dans le sud de la Loire-Atlantique (tabl. 4 ; fig. 10), à l'exception d'un unique exemplaire sur le site de la Butte-aux-Pierres (SaintJoachim : fig. 11, n 22 ), où s'agglomèrent différentes composantes chronologiques. La présence d'un seul spécimen sur ce site, tout comme à La Miquenaie (Saint-Michel-etChanveaux, Maine-et-Loire : ibid., $\mathrm{n}^{\circ}$ 23), second site du corpus à en posséder au nord de la Loire, invite à modérer le statut d'armature finie et désirée. Le caractère effilé de ce sous-type local segmente le corpus en deux grands groupes et souligne la possible flexibilité de sa morphométrie. La Loire-Atlantique et la Vendée ne possèdent que des pointes assez trapues. Le groupe des pointes effilées s'illustre par les sites de la Miquenaie (Gouraud et Tessier, 1998; fig. 11, $\mathrm{n}^{\circ} 23$ ), de la Pierre-Saint-Louis (ibid., nº 14, 15), de l'Abri des Rocs (Bellefonds; ibid., $\mathrm{n}^{\circ}$ 16) et de la grotte de Gioux (Chauvigny, Vienne; ibid., $\mathrm{n}^{\circ}$ 17). L'indice d'allongement atteint son taux maximal dans les deux grottes viennoises.

Concluons la caractérisation de cette zone sur la spécificité des triangles. Habituellement reconnus comme type ubiquiste, ils ne constituent qu'environ un tiers du carquois ligérien contre un peu moins de la moitié du vendéen et deux tiers du charentais.

\section{Le carquois des sites vendéens}

Ce fonds microlithique s'approche de celui des gisements de la Loire-Atlantique par un faible taux de lamelle à bord(s) abattu(s) et un goût assez prononcé pour les pointes à base naturelle. Il possède cependant une composition qui lui est propre : alors que dans les deux zones présentées auparavant une préférence pour des types d'armatures précis s'affiche, on a ici affaire à un certain équilibre entre les pointes à base naturelle, celles à base retouchée, les triangles isocèles et les spécimens scalènes. Hormis la faible présence des pointes de Chaville, peu d'autres particularités locales majeures ont été décelées.

L'est vendéen s'est démarqué lors de l'analyse de répartitions spatiales des différents sous-types de triangles scalènes : on y retrouve seulement un sixième des modèles observés dans le Centre-Ouest. Les préférences vont aux sous-types sans piquant-trièdre apparent, au troisième côté non retouché et aux troncatures rectilignes, la grande troncature pouvant également - mais dans une moindre mesure - être convexe. Pour les triangles scalènes, il semble donc que ces tailleurs aient opté pour des choix plus stricts que dans les zones alentour. Il peut également être intéressant de noter une large supériorité des pointes à base naturelle et troncature concave en Vendée, alors qu'inversement celles à troncature convexe s'y trouvent sous-représentées par rapport aux autres départements. Cependant la distribution spatiale dispersée de ces sous-types n'argumente pas en faveur d'un particularisme local fortement marqué.

\section{Aperçu des assemblages des départements de la Sarthe, du Maine-et-Loire, des Deux-Sèvres et de la Vienne}

Sur ces aires géographiques, un rapprochement avec un quelconque faciès typologique semble vain puisque peu d'assemblages y ont été repérés; quelques tendances peuvent tout de même être mentionnées. Les industries des sites des trois premiers départements se comportent globalement comme celles des gisements des Pays-de-la-Loire. Des interactions particulières entre le nord de la France et les sites de la Sarthe semblent s'afficher à travers la prédominance de pointes à base naturelle et un statut parfois important des segments de cercle. Ceux-ci sont également bien représentés en Maineet-Loire dans les gisements au nord de la Loire. La pointe de la Majoire permet de mettre en évidence des contacts entre le sud de la Loire, l'est de la vallée de la Chère, le sud de la vallée de la Sèvre nantaise ou la vallée de la Vienne. Les carquois de cette dernière tendent à rejoindre tantôt ceux du groupe stylistique Loire-Atlantique/Vendée (notamment par la très grande discrétion des lamelles à bord(s) abattu(s) ou des triangles de Montclus), tantôt ceux des Charentes comme l'illustre la faible part des pointes à base naturelle.

\section{Approche du faciès « $C$ »}

La caractérisation stylistique de ce faciès est restreinte tout d'abord par le fait que moins de $15 \%$ des sites recensés s'y rattachent. Elle pose également problème compte tenu des méthodes de ramassage du mobilier qui ne sont généralement pas adaptées au phénomène d'hypermicrolithisation des armatures qui lui est propre. Ajoutons à cela la forte fragmentation des pièces, peut-être liée aux petites mensurations des armatures, qui empêche l'identification de plus d'un quart des microlithes. L'étude de la partie septentrionale du Centre-Ouest se restreint à quelques simples constatations, telle l'absence de lamelles à bord(s) abattu(s). Pour les triangles et les pointes, hormis le changement de proportions des sous-types, leur morphologie est équivalente à celle précédemment décrite (cf. supra). Notons que les pointes 
fusiformes et segments de cercle représentent environ un sixième du fonds microlithique de la Loire-Atlantique.

Les Charentes peuvent être mieux caractérisées, essentiellement grâce au site de la Grange à Surgères. Les microlithes segmentiformes ne semblent pas faire partie de la palette des tailleurs. Leur préférence va, dans plus de la moitié des cas, aux triangles et favorise surtout les scalènes miniatures allongés et effilés. Les pointes à base retouchée forment la deuxième catégorie d'armatures majoritaire. Leur taux, proche de $20 \%$, se différencie fortement de celui des industries montclusiennes ${ }^{9}$ et évoque plus ce qui s'observe au nord du Centre-Ouest. Les spécimens à base naturelle, qui sont légèrement moins nombreux que les précédentes, participent à cette même tendance mais dans une moindre mesure. La présence de lamelles à bord(s) abattu(s) n'est que clairsemée; sur le site de la Grange par exemple, des armatures fracturées pourraient s'intégrer à ce type mais aucune preuve n'est formelle (fig. $6, \mathrm{n}^{\circ} 45,46$ ). Les deux sous-types caractéristiques du Sauveterrien sont peu marqués : on ne dénombre aucune pointe présentant tous les critères pour être rattachée au type de Sauveterre, alors que des triangles de Montclus garnissent quelques carquois (fig. 8). Mais ceux-ci, à classer essentiellement dans les modèles apparentés, sont insuffisamment nombreux pour être réellement représentatifs.

\section{Centre-Ouest et Grand-Ouest : LIENS STYLISTIQUES ET INTERACTIONS}

L'appréciation de la nature des différentes interactions au sein du Grand-Ouest est d'autant plus difficile que les références régionales ne se trouvent pas à proximité immédiate du Centre-Ouest.

\section{Évaluation des caractéristiques des faciès « $A$ » et « $B$ » en Centre-Ouest}

\section{Versants septentrional et oriental}

Des tendances similaires dans le choix des microlithes semblent s'afficher entre le Centre-Ouest et les communautés voisines septentrionales, avec notamment un faible recours aux segments de cercle (fig. 12). Les affinités entre les armatures du Centre-Ouest et celles du sud-est de la Bretagne (Morbihan, vallée de la Vilaine) semblent plus développées qu’avec celles des communautés plus septentrionales (nord-Bretagne, nordCotentin : Marchand et al., 1998). La préférence accordée aux pointes et triangles s'illustre notamment par les indices, certes ténus, de Saint-Melaine et du Prieuré (Pléchâtel) ou de Cojou

9. Le Montclusien correspond au stade moyen du Sauveterrien (Barbaza et al., 1991).
(Saint-Just), en Ille-et-Vilaine. Les mêmes types d'arguments ont servi à la mise en parallèle des industries du Centre-Ouest, de l'Indre et de la rive gauche du cours moyen de la Loire (Audoux et Thévenin, 1995; Bazin et al., 1995; Gouraud, 1995) même si, dans les départements présentement étudiés, le taux de segments de cercle est encore moins important. Des affinités plus affirmées se dessineraient entre les sites des Paysde-la-Loire et ceux de Moulin-du-Saule (Autry-le-Châtel, Loiret) ou de Montierchaume (Indre) par exemple, à travers la multiplicité des pointes à base retouchée concave (Audoux et Thévenin 1995; Bazin et al., 1995). Enfin, la présence de pointes de Chaville, plus marquée dans les industries des sites de la Loire-Atlantique, pourrait évoquer, comme l'indique la position géographique du site éponyme dans les Hautsde-Seine, de probables contacts interculturels avec le sud du Tardenois. Cependant, ces armatures sont exceptionnelles, voire absentes, sur les franges septentrionale et orientale du Centre-Ouest.

\section{Un Sauveterrien envahissant?}

Le Sauveterrien est la seule entité technique bien définie en contact immédiat avec le Centre-Ouest, ce qui a parfois eu comme effet de complètement gommer l'existence parallèle d'autres interactions et de caractéristiques propres à ce dernier.

Si l'on s'en tient à la majorité des schémas traditionnels, le Centre-Ouest est sous influence sauveterrienne ou même assimilé à ce techno-complexe. Le recours à ce type d'hypothèse diffusionniste, avec un centre rayonnant sur sa périphérie, se justifie mal pour le premier Mésolithique compte tenu du peu d'indices à disposition et de la défaillance du système chronologique : cette hypothèse serait à revoir au profit d'un modèle "d'entités techniques en interactions réciproques " (Blanchet et al., 2006). Les études concluant à un éventuel rattachement du Centre-Ouest à ce techno-complexe se sont souvent basées sur la seule présence de deux sous-types caractéristiques du Sauveterrien : les triangles de Montclus et les pointes de Sauveterre. Ainsi, la découverte d'un triangle de Montclus - associé à deux spécimens apparentés - et d'une pseudo-pointe de Sauveterre sur les sites du Bois des Jarries (Saint-Mars-la-Réorthe, Vendée : fig. 6, $n^{\circ} 52$ ), de la Majoire $C\left(n^{\circ} 51\right)$ et de la Garne $\left(n^{\circ} 50\right)$ a parfois suffi à l'évocation de fortes affinités sauveterriennes ou du rôle moteur de cette entité (Gouraud et al., 1993; Valdeyron, 1994; Gouraud et al., 1996).

La composition microlithique des carquois du CentreOuest se distingue en fait de celle du techno-complexe méridional par la grande discrétion des pointes de Sauveterre et des triangles de Montclus ou par la proportion assez importante de pointes à base retouchée, type rare ou absent des gisements de la France méridionale. Le piquant-trièdre par- 

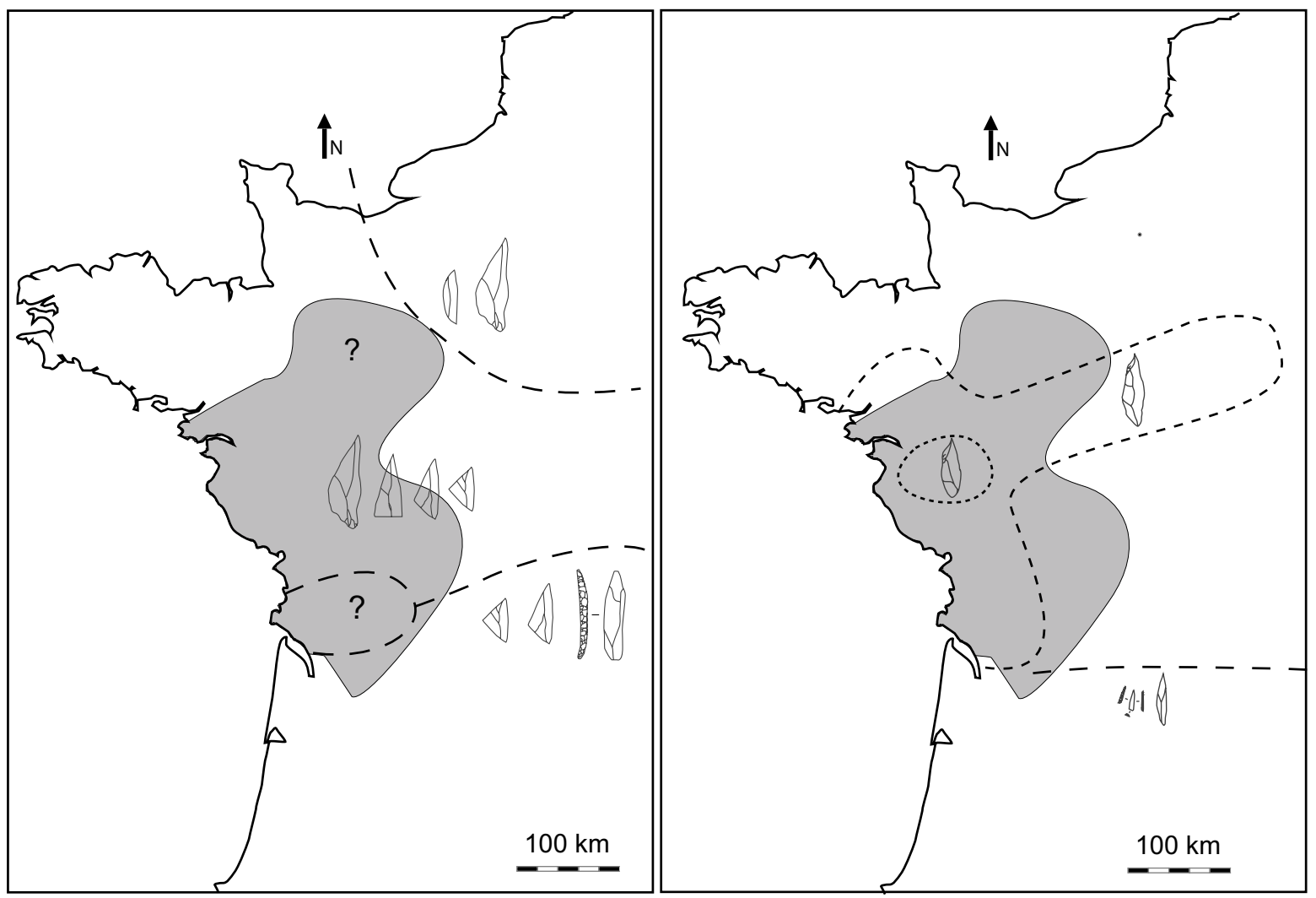

Aires de répartitions des principaux groupements de types d'armatures
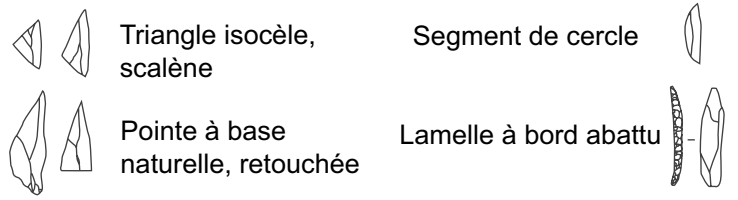

Aires de concentrations des principaux sous-types d'armatures
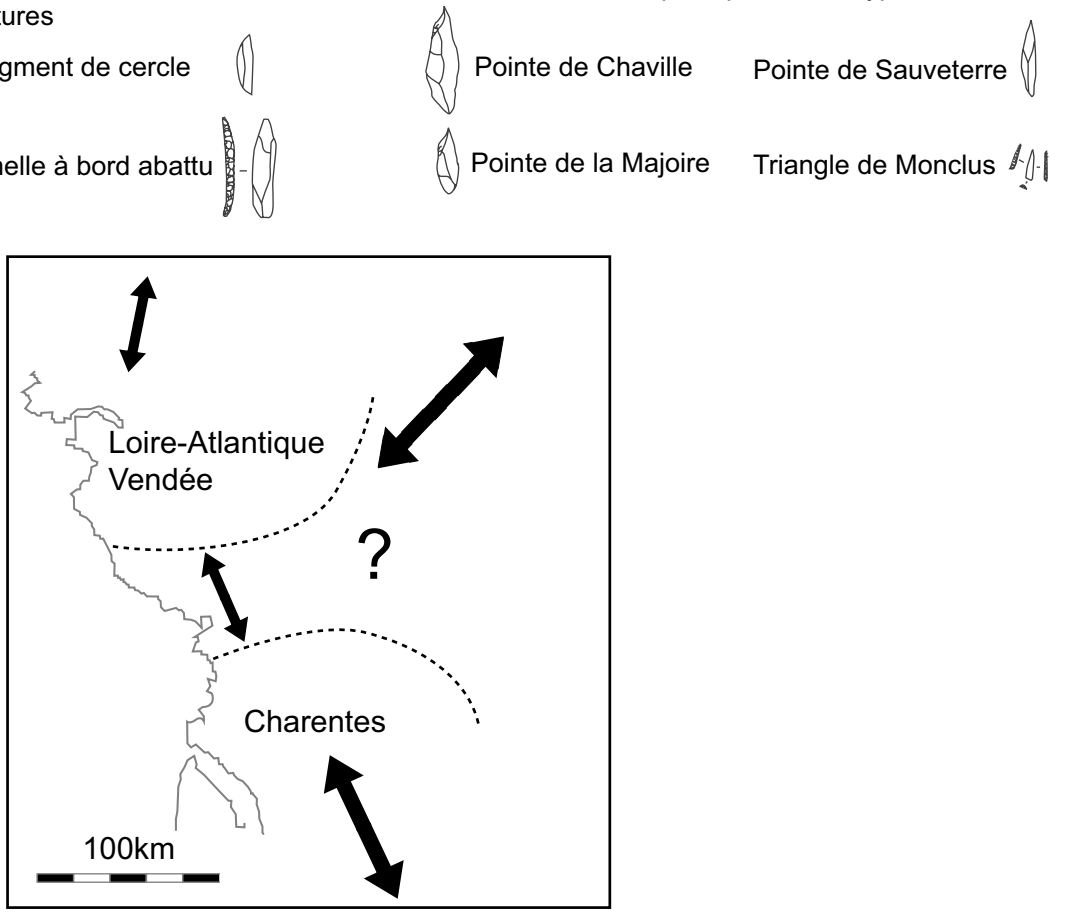

Essai de synthèse des particularismes stylistiques en Centre-Ouest et des interactions au sein de cette zone et avec celles avoisinantes

Figure 12 : Représentation spatiale des associations d'armatures pour les faciès « $A$ » et « $B$ » (zone d'étude est en grisé), et carte de synthèse.

Figure 12: Spatial representation of arrowheads associations for facies " $A$ " and " $B$ " (studied area is shaded), and synthesis map. 
ticipe aussi à cette distinction au niveau des pointes : très rarement observable en contexte sauveterrien, sa conservation est visible dans les carquois du Centre-Ouest. Il en est de même pour la proportion marquée de triangles scalènes à petite troncature concave, sous-type moins représenté - voire quasiment absent - dans le Sauveterrien.

Alors que l'observation seule de la répartition spatiale des triangles de Montclus et des pointes de Sauveterre aurait servi l'hypothèse d'une plus grande proximité du Sauveterrien avec les Pays-de-la-Loire qu'avec les Charentes, l'analyse du fonds microlithique total du Centre-Ouest montre une tendance inverse. En effet, les sites de Loire-Atlantique et de Vendée, $\mathrm{y}$ compris ceux pour lesquels des indices sauveterriens ont précédemment été annoncés, présentent des critères témoignant d'une interaction moins marquée avec le sud-ouest de la France et mettent en évidence d'autres contacts et des particularismes locaux. À ce titre, on peut souligner la place de choix accordée aux pointes à base retouchée, la fréquence de la concavité de leur base (60\% aux Bois des Jarries, $75 \%$ à La Majoire C, 87,5 \% à La Garne) et l'existence marquée de la pointe de Chaville. Selon G. Gouraud et M. Tessier (Gouraud, 1996; Gouraud et Tessier, 1998), l'armature de la Majoire tiendrait le rôle de la pointe de Sauveterre en Centre-Ouest, d'où son statut de « témoin discret d'influences sauveterriennes en Basse-Loire ". La ressemblance entre ces deux sous-types ne repose que sur leur allure fusiforme, qui est d'ailleurs beaucoup plus régulière sur les pointes typiques du techno-complexe méridional. Les choix divergent réellement d'un point de vue dimensionnel. Rapprocher strictement ces deux types d'armatures minimise l'importance accordée à la conservation du piquant-trièdre, choix qui rappelle la pointe de Chaville et traduit des interactions entre le domaine sud-ligérien du Centre-Ouest et les entités techniques plus septentrionales. Les pointes de la Majoire ne sont associées à celles de Sauveterre qu’à Bellefonds, mais dans près de $44 \%$ des gisements présentant les premières de ces armatures on trouve des pointes de Chaville. Ce fait pourrait souligner la primauté de la conservation du piquant-trièdre pour le sous-type de la Majoire.

Ce travail indique que les Charentes s'affichent dans une tendance plus méridionale. En témoignent les lamelles à bord(s) abattu(s) qui constituent un élément récurrent des industries sauveterriennes, mais qui conservent un statut numériquement inférieur dans les Charentes. Ce lien avec le techno-complexe méridional, d'où provient probablement le concept de ce type de microlithes, n'est pas exclusif à en voir leurs divergences. Les triangles de Montclus n'appartiennent pas au fonds microlithique des Charentes à l'exception du site de La Pierre-Saint-Louis, seul gisement charentais d'ailleurs où la collection comporte des pointes de la Majoire. Alors que les pointes de Sauveterre n'apparaissent pas non plus dans ces sites, les pointes de Chaville garnissent leur carquois dans un cas sur deux. La fréquence de ces dernières irait à l'encontre d'une tradition sauveterrienne la plus stricte (Valdeyron, 1994). De même, le taux de pointes à base retouchée est largement supérieur à celui de l'industrie sauveterrienne.

De récents travaux remettent en question l'emprise du Sauveterrien sur la façade atlantique. L'idée d'un éventuel «Sauveterrien atlantique " (Roussot-Larroque, 2000) repose sur l'observation des pointes des sites d'Aquitaine occidentale, dont certaines à base concave évoquent les exemplaires ligériens. La proportion des microburins est également évoquée comme facteur de différenciation avec le Sauveterrien. Le raisonnement d'A. Thévenin (2005) ajoute l'observation de l'angle interne des troncatures des triangles et de leur distance de fixation. Il rattache les spécimens étroits au Sauveterrien et les modèles larges au " Mésolithique ancien ligérien ». En joignant à ce dernier les sites comportant des éléments des deux composantes culturelles, il évoque l'existence d'un groupe technique se distinguant du Sauveterrien : le "Mésolithique ancien atlantico-ligérien » localisé du Sud-nantais aux Pyrénées jusqu’à $150 \mathrm{~km}$ de la façade atlantique. Il fait de la répartition des couteaux à encoches basilaires un argument confirmant son hypothèse. Il semble qu'il faille moduler et préciser quelque peu les normes précédemment utilisées. Celles proposées pour les triangles ont été appliquées à l'ensemble des sites présentement étudiés : les résultats obtenus divergent de ceux énoncés auparavant puisque plusieurs corpus, jusqu'au nord même de la Loire, ne comportent que des exemplaires étroits. La répartition des couteaux précités ne semble pas permettre de parler plus formellement d'entité atlantique. En effet, en dehors de la concentration du Centre-Ouest, on en recense certes sur le site de Rouffignac (Dordogne) mais aussi sur la rive gauche de la Loire moyenne au Moulin-du-Saule (Autryle-Châtel, Loiret), ou encore à Ruffey-sur-Seille dans le Jura (Gouraud et Thévenin, 2000). Ces couteaux ne viennent donc pas étayer l'hypothèse d'une seule et même entité sur la façade atlantique de la France mais expriment plutôt des contacts à une plus large échelle entre différentes entités. Le développement de cette problématique en précisant et multipliant les critères d'étude permettra peut-être de voir se dessiner plus formellement cette entité technique. L'alternance de modèles unifiant ou scindant le Sauveterrien montre bien en tout cas que sa définition est plus complexe qu'il n'y parait.

\section{Entités stylistiques du Centre-Ouest}

Dans le Centre-Ouest, il semble que nous ayons affaire au moins à deux entités stylistiques (fig. 13), qui entretiennent des rapports plus ou moins prononcés entre elles et avec les entités techniques septentrionales et méridionales.

La première, localisée en Loire-Atlantique et au nord de la Vendée, est caractérisée par la rareté des segments de cercle, 


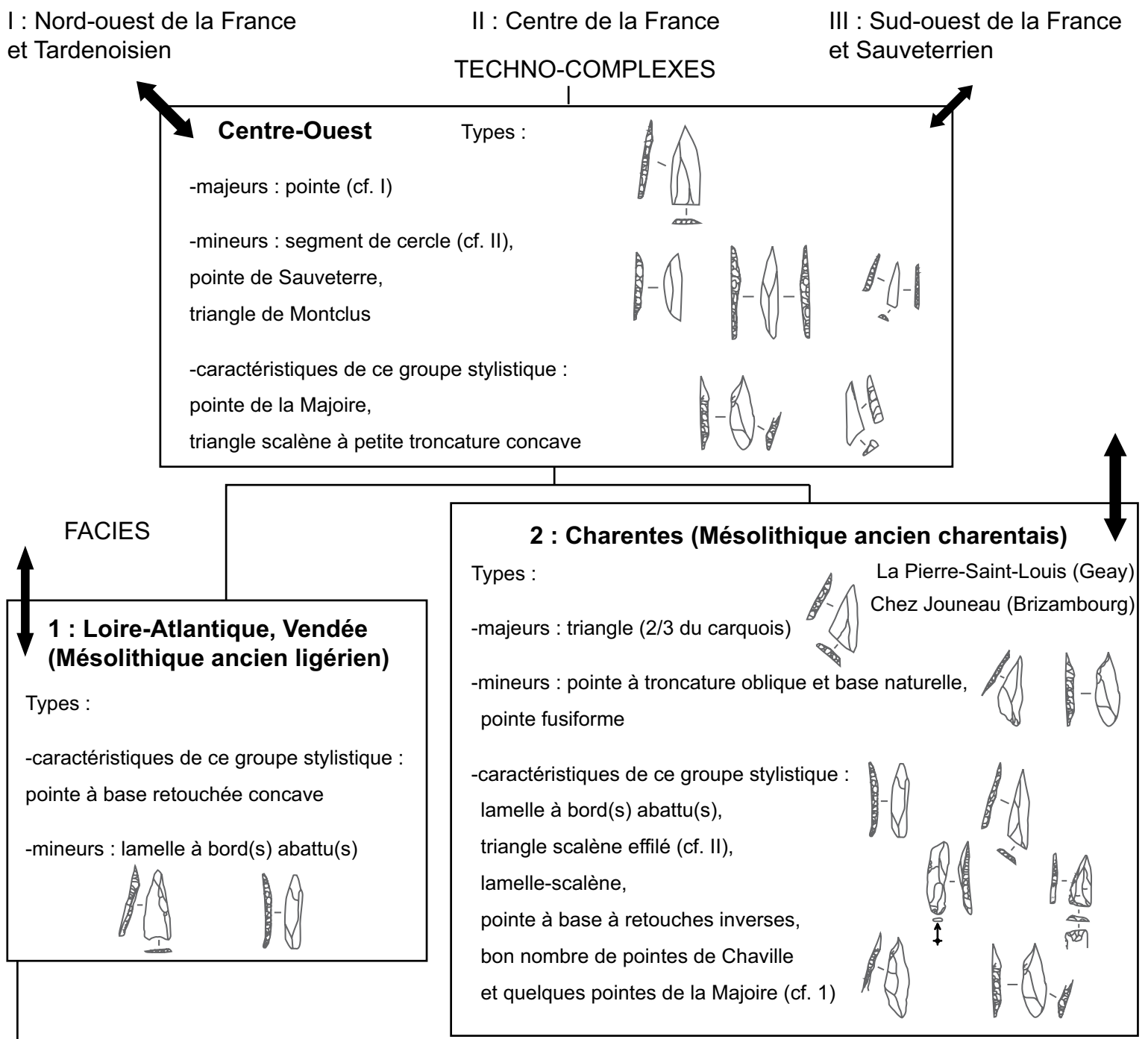

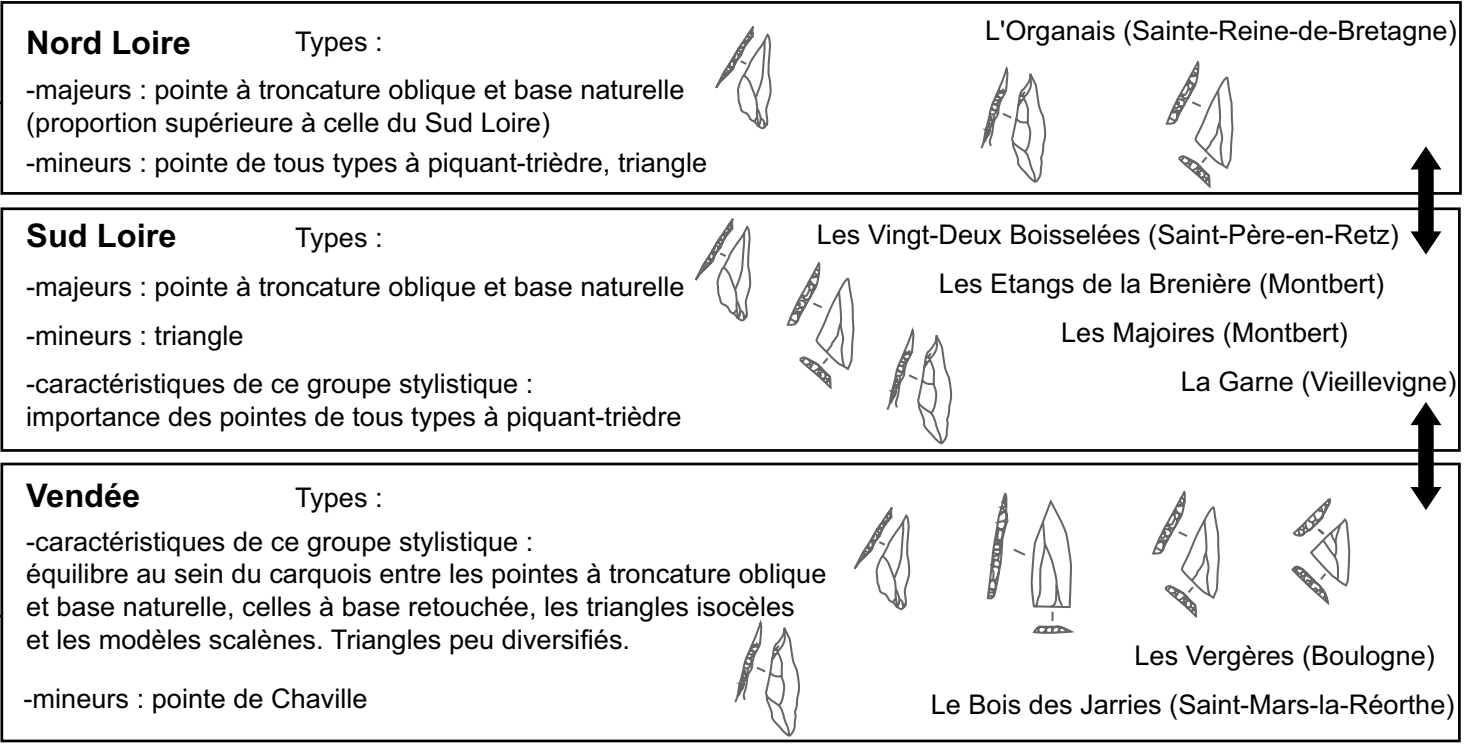

Figure 13 : Synthèse sur les industries du Centre-Ouest pour les faciès « $A$ » et « B ».

Figure 13: Synthesis of Centre-West France industries (facies " $A$ " and "B"). 
des lamelles à bord(s) abattu(s) et des sous-types caractéristiques du Sauveterrien, ceci au bénéfice de tous types de pointes et notamment de celles à base retouchée concave ou de la Majoire. Alors que les sites vendéens s'individualisent par un carquois équilibré, les industries proches de la Loire ont pour singularité stylistique une prédominance de pointes à base naturelle, dont celles de Chaville. Les spécificités décrites participent à l'appréciation du "Mésolithique ancien ligérien " (Valdeyron, 1994) et en complètent la définition.

La seconde entité stylistique, dont le siège serait les Charentes, correspond à un fonds microlithique largement dominé par les triangles mais faisant également bonne place aux pointes à base retouchée, et dans une moindre mesure aux lamelles à bord(s) abattu(s); on ne peut pas en dire autant des triangles de Montclus ni des autres types de pointes (de la Majoire, de Sauveterre) - même si celles de Chaville s'imposent tout de même en assez bonne quantité. Par mimétisme, nous pourrions parler de "Mésolithique ancien charentais ", cette appellation n'ayant une valeur qu'en terme stylistique. Dans les études à venir il faudra observer si les spécificités stylistiques ainsi énoncées concernent des sites extérieurs à cette zone. La découverte d'indices dans l'intervalle de $90 \mathrm{~km}$ entre les sites les plus méridionaux des Pays-de-la-Loire et les plus septentrionaux des Charentes faciliterait la compréhension de l'imbrication de ces deux zones.

Ces deux entités stylistiques, qui pourraient s'apparenter à des faciès culturels non hermétiques, ne relèvent pas de traditions totalement similaires. Les particularismes locaux bien affirmés associés à des caractères communs, qui ne peuvent pas être niés et qui traduisent parfois des contacts avec des groupes humains externes au Centre-Ouest, semblent concorder vers un modèle de larges réseaux d'interactions bien développés. Il semble difficile d'éclaircir de façon plus précise la nature des relations entre les communautés humaines, aussi bien au sein du Centre-Ouest qu'à l'échelle du Grand-Ouest, à partir des seules associations d'armatures mises au jour. La tripartition stylistique de l'ouest de la France (fig. 12), proche d'un point de vue formel de modèles traditionnels mais fondée sur des critères différents, intégrerait la Loire-Atlantique et la Vendée à un éventuel techno-complexe faisant la part belle aux pointes mais aussi aux triangles et qui resterait à définir. Il serait en revanche plus présomptueux de rattacher formellement le groupe stylistique charentais à un techno-complexe donné. Son degré d'affinité avec le Sauveterrien ne contrebalance pas des divergences trop marquées pour parler d'appartenance à un seul et même techno-complexe industriel. L'apparente mixité du fonds microlithique de ce faciès stylistique forme une nébuleuse difficile à décrypter, peut-être à expliquer par un complexe jeu d'interactions avec les sphères du centre et du sud de la France.

\section{Faciès « $\mathrm{C}$ » : Grand-Ouest unifié ou diversifié?}

L'observation des carquois du Grand-Ouest a souvent privilégié l'impact de la composante méridionale jusqu'à la pointe finistérienne. Le groupe de Bertheaume a ainsi parfois été rattaché à la sphère sauveterrienne par ses "lamelles bertheaume ", qui ont parfois été associées aux pointes sauveterriennes (Kayser et al., 1990), et pour ses triangles scalènes étroits. Ces derniers s'apparentent parfois à des triangles de Montclus, comme à Toul-an-Naouc'h (Plougoulm). Les corpus des entités septentrionales voisines (nord-Bretagne, nord-Cotentin) s'en démarquent avec des armatures d'un calibre plus important (Ghesquière et al., 2003). Dans les gisements de la Manche, à l'image de Roc de Gîte (Auderville), les lamelles à bord(s) abattu(s), type dont le concept initial semble à tendance méridionale, sont majoritaires, suivies de pointes à troncature très obliques (ibid.). Le Centre-Ouest s'impose entre le Nord-Ouest et le Sauveterrien montclusien, ne favorisant pas l'idée d'une extension du techno-complexe méridional jusqu'à la pointe de Bretagne (Blanchet et al., 2006). La présente étude ne peut que souligner ces propos. Malgré le faible nombre de sites rattachables au faciès " $C$ », une tonalité plus diversifiée que purement méridionale se dessine dans les choix stylistiques. Outre l'importance accordée aux pointes, dont celles à base concave - aussi évoquées pour le sud-Bretagne (Marchand, 2005), une faible part voire une quasi-absence de lamelles à bord(s) abattu(s) est notable (cf. supra.). La très faible représentation des deux sous-types caractéristiques du Sauveterrien semble également assez évocatrice. Il ne semble donc pas que l'on puisse purement et simplement attribuer les Charentes - et encore moins en l'état actuel des données le nord du Centre-Ouest - au Montclusien, les interactions décelées n'étant pas exclusives. L'existence de réseaux d'interactions entre des groupes - ni dissociés ni totalement apparentés - est tout à fait probable, comme pour les faciès précédemment étudiés. Les outils communs et les modes de débitage feraient office, dans le faciès " $\mathrm{C}$ ", de trait d'union entre les différentes entités individualisées par la spécificité de leur association d'armatures (Blanchet et al., 2006).

\section{Proposition d'Un MOdèle d’organisation DES TERRITOIRES STYLISTIQUES DU PREMIER Mésolithique dans le Centre-Ouest de la France}

L'hypothèse du rôle moteur du Sauveterrien sur les entités stylistiques et culturelles du Centre-Ouest au premier Mésolithique a majoritairement été privilégiée dans les sché- 
mas traditionnels suite à cette première combinaison de l'ensemble des données sur les armatures du Centre-Ouest; il semble nécessaire de rétablir l'importance à accorder aux interactions avec d'autres régions ainsi qu'aux particularismes locaux.

Les faciès " $A$ " et « $B$ " associent deux entités stylistiques distinctes mais perméables, aussi bien entre elles qu'avec les entités environnantes.

- Celle de la Loire-Atlantique et de la Vendée (Mésolithique ancien ligérien) pourrait être scindée en un groupe stylistique prédominé par les pointes à base naturelle et un autre au carquois équilibré entre pointes et triangles; tous deux forment un faciès stylistique dépourvu de segments de cercle et de lamelles à bord(s) abattu(s), ceci au profit des pointes à base retouchée concave ou de la Majoire.

- La spécificité de l'entité stylistique des Charentes (Mésolithique ancien charentais) peut se résumer à un fonds microlithique basé sur le trio triangles/pointes à base retouchée/lamelles à bord(s) abattu(s). L'association de ce faciès à un techno-complexe donné pose pour l'instant problème et la nature de ses relations avec le Sauveterrien est à éclaircir.

Au faciès " $\mathrm{C}$ ", on ne peut proposer qu'une approche stylistique pour le sud du Centre-Ouest : alors que les armatures segmentiformes se font discrètes, on note un goût très prononcé pour les triangles scalènes allongés. À côté des pointes à base retouchée, les lamelles à bord(s) abattu(s) et triangles de Montclus font pâle figure.

Pour définir les groupes culturels du début de l'Holocène en Centre-Ouest, il faut prendre en compte des spécificités stylistiques locales à régionales au sein des faciès mais aussi des réseaux d'interactions réciproques plus ou moins prononcées qui sont loin d'être orientées exclusivement vers une entité technique, qu'elle soit sauveterrienne ou non.

L'état actuel des recherches sur le Centre-Ouest se résume à des mentions variées d'éléments stylistiques et de rares études technologiques. Il ne permet donc de réaliser qu'une analyse typologique, qui ne reste qu'une étape préliminaire dans l'approche des populations mésolithiques en jeu. L'optique est désormais de réaliser une étude plus globale de l'ensemble des indices à disposition pour avoir une meilleure vision de ces sociétés de chasseurs-cueilleurs ${ }^{10}$. La validité des « territoires » stylistiques proposés est ainsi testée et l'appréciation du territoire identitaire complétée grâce à la confrontation à d'autres types de marqueurs. L'étude des territoires d'acquisition des matières premières taillables offre en effet une image du territoire de parcours - ou territoire d'itinérance (Gouletquer, 1991), traduisant une autre dimension géo-

10. Les premiers groupes mésolithiques dans l'Ouest de la France (10000 -6000 avant notre ère) : territoires stylistiques et organisation économique: S. Michel, doctorat en cours sous la direction de Grégor Marchand et Nathalie Molines (UMR 6566). graphique. La compréhension de la gestion de l'espace et de son organisation économique implique une réflexion sur le statut des habitats et la saisonnalité des déplacements. L'approche technologique de tous les vestiges lithiques, visant à restituer les chaînes opératoires de production et d'utilisation, contribue également à cette diversification des marqueurs identitaires.

\section{Bibliographie}

Audoux, D. et Thévenin, A., 1995 - L'Épipaléolithique et le Mésolithique du département de l'Indre, in Thévenin, A. (dir.), Épipaléolithique et Mésolithique du Sénonais et des régions voisines (actes du colloque de Passy, 20-21 novembre 1993), Cahier 2. Sens, Société archéologique de Sens, p. 133-149.

Barbaza, M., Valdeyron, N., Andre, J., Briois, S., Martin, H., Philibert, S., Allios, D. et Lignon, F., 1991 - Fontfaurès en Quercy. Contribution à l'étude du Sauveterrien, Toulouse, École des hautes études en sciences sociales (Archives d'Écologie préhistorique, 11), 269 p.

BARTH, F., 1969 - Ethnic groups and boundaries. The social organisation of Culture difference, Boston, Little, Brown, 153 p.

Bazin, P., Halley, J.-P. et ThÉVEnin, A., 1995 - Les stations mésolithiques d'Autry-le-Châtel (Loiret), in ThÉvenin, A. (dir.), Épipaléolithique et Mésolithique entre Seine et Rhin (table ronde d'Ancerville, 1989), Besançon, Annales littéraires de l'université de Besançon, p. 181-212.

Blanchet, S., Kayser, O., Marchand, G. et Yven, E., 2006 - Le Mésolithique moyen en Finistère : de nouvelles datations pour le groupe de Bertheaume, Bulletin de la Société préhistorique française, 103, p. 507-517.

Boissinot, P., 1998 - Que faire de l'identité avec les seules méthodes de l'archéologie?, in D'AnnA, A. et Binder, B. (dir.) - Production et identité culturelle, actualité de la recherche (Rencontres méridionales de la Préhistoire récente, actes de la $2^{\mathrm{e}}$ session : Arles 1996). Antibes, APDCA, p. 17-25.

Bonte, P. et IZARD, M., 1991 - Dictionnaire de l'ethnologie et de l'anthropologie. Paris, Presses universitaires de France, 842 p.

Brunet, R., Ferras, R. et Théry, H., 1992 - Les mots de la géographie, dictionnaire critique, Paris, Reclus/La Documentation Française, 518 p.

Collignon, B., 1996 - Les Inuits : ce qu'ils savent du territoire. Paris, L'Harmattan, 254 p.

Cupillard, C. et Richard, A., 1999 - Les derniers chasseurs cueilleurs du massifjurassien et de ses marges (13000-5500 avant Jésus-Christ), Lons-le-Saunier, Centre Jurassien du Patrimoine, $229 \mathrm{p}$.

Demars, P.-Y., 1986 - L'interprétation des industries lithiques et leur rapport avec la culture, Paris, Université de Paris 1/Centre de Recherches préhistoriques (Cahier 10), p. 87-94. 
Dietler, M. et Herbich, I., 1994 - Habitus et reproduction sociales des techniques. L'intelligence du style en archéologie et en ethno-archéologie, in Latour, B. et Lemonnier, P. (dir.), De la Préhistoire aux missiles balistiques. L'intelligence sociale des techniques, Paris, La Découverte, p. 204-227.

Ducroce, T., 2001 - Le Mésolithique du bassin de la Somme : insertion dans un cadre morpho-stratigraphique, environnemental et chronoculturel, Villeneuve-d'Ascq, Université des Sciences et Technologies de Lille (Centre d'Études et de Recherches préhistoriques), $248 \mathrm{p}$.

Dugast, C., Gouraud, G. et Jauneau, J.-M., 2000 - La station mésolithique des Vergères à Boulogne (Vendée), Revue archéologique de l'Ouest, 17, p. 37-72.

Foucher, P. et SAN Juan, C., 2004 - The tardiglacial and initial Holocene on the Charente-Maritime and Vendée coast, in Gonzalez Morales, M. and Clark, G.-A. (ed.), The Mesolithic of the Atlantic Façade (Proceedings of the Santander Symposium), Tucson, Arizona State University (Anthropological Research Papers, 55), p. 69-87.

GEEM, 1969 - Épipaléolithique-Mésolithique, les microlithes géométriques, Bulletin de la Société préhistorique française, 66, p. 355-366.

GEEM, 1972 - Épipaléolithique-Mésolithique, les armatures non géométriques, Bulletin de la Société préhistorique française, 69, p. 364-375.

Giddens, A., 1987 - La constitution de la société, Paris, Presses universitaires de France, $474 \mathrm{p}$.

Giligny, F. et Sidi-MaAmar, H., 1990 - Simulation archéologique à partir de l'étude ethno-archéologique des flèches de Ye Ineri, Irian-Jaya (Indonésie), Histoire et Mesure, 5, p. 145-162.

Ghesquière, E., Lefèvre, P. et Marcigny, C., 2003 - Le Mésolithique moyen du nord-Cotentin (Manche), Bulletin de la Société préhistorique française, 100, p. 649-670.

GouletQuer, P., 1979 - Préhistoire du futur, Saint-Hernin, éditions Bretagne, $112 \mathrm{p}$.

Gouletquer, P., 1991 - Les problèmes posés par le Mésolithique de Basse-Bretagne : les moyens de les résoudre. Mésolithique et néolithisation en France et dans les régions limitrophes $\left(113^{\mathrm{e}}\right.$ Congrès national des Sociétés savantes, Strasbourg 1988). Paris, CTHS, p. 177-196.

Gouraud, G., 1987 - La pointe de la Majoire. Revue archéologique de l'Ouest, 4, p. 35-36.

Gouraud, G., 1995 - Introduction au Préboréal du CentreOuest, in ThÉvenin, A. (dir.), Épipaléolithique et Mésolithique $d u$ Sénonais et des régions voisines (Actes du colloque de Passy, 20-21 novembre 1993, cahier 2). Sens, Société archéologique de Sens, p. 181-184.

Gouraud, G., 1996 - Le microlithisme de la Pierre-Saint-Louis à Geay (Charente-Maritime) dans le cadre du Mésolithique régional (Mémoire de DEA), Toulouse, Université de Toulouse-Le Mirail et École des hautes études en sciences sociales, 228 p.
Gouraud, G., Dugast, C., Jauneau, J.-M. et Cousseau, B., 1993 - La station mésolithique de La Garne à Vieillevigne, Bulletin du Groupe vendéen d'Études préhistoriques, 29, p. 1-19.

Gouraud, G., Dugast, C. et Jauneau, J.-M., 1996 - Le Mésolithique des Majoires à Montbert (Loire-Atlantique), IILa station C, Revue archéologique de l'Ouest, 13, p. 31-50.

Gouraud, G., et Tessier, M., 1998 - La pointe de la Majoire et les pointes fusiformes à piquant-trièdre en Centre-Ouest, Bulletin de la Société nantaise de Préhistoire, 21, p. 5-19.

Gouraud, G. et Marchand, G., 1999 - Typologie des armatures dans l'ouest de la France, Bulletin du Groupe vendéen d'Études préhistoriques, 35, p. 1-20.

Gouraud, G. et Thévenin, A., 2000 - Les couteaux à encoches basilaires, Revue archéologique de l'Ouest, 17, p. 87-99.

Kayser, O., Le Goff, J.-C. et Roué, D., 1990 -Le site mésolithique de Toul-an-Naouc'h (Plougoulm, Finistère), Revue archéologique de l'Ouest, 7, p. 23-29.

KozlowsKi, S.-K., 1975 - Cultural differenciation of Europe from 10th to 5th Millennium B.C., Warsaw University Press, $259 \mathrm{p}$.

Kozlowski, S.-K., 2003 - E pluribus unum? Regards sur l'Europe mésolithique, in Desbrosse, R. et Thévenin, A. (dir.), Préhistoire en Europe, des origines à l'Âge du Bronze, Paris, CTHS, p. 293-300.

Lemercier, O., 2000 - Espace culturel, territoire et terroir : approches spatiales des groupes campaniformes récent dans le sud-est de la France, in Leduc, M., Valdeyron, N. et VAquer, J. (dir.), Sociétés et Espaces, (Rencontres méridionales de Préhistoire récente, $3^{\mathrm{e}}$ session, Toulouse 1998), Toulouse, Archives d'Écologie préhistorique, p. 177-186.

Lemonnier, P., 1987 - Le sens des flèches. Culture matérielle et identité ethnique chez les Anga de Nouvelle-Guinée, in Koechlin, B., Sigaut, F., Thomas, J. et Toffin, G. (dir.), De la voûte céleste au terroir, du jardin au foyer. Mosaïques sociographiques, Paris, École des hautes Études en Sciences sociales, p. 573-595.

Marchand, G., 2000 - Facteurs de variabilité des systèmes lithiques au Mésolithique récent et final dans l'ouest de la France, in Crotti, P. (dir.), Méso' 97 (Table ronde sur l'Épipaléolithique et le Mésolithique, Lausanne 1997), Cahiers de l'Archéologie romande, 81, p. 37-48.

MarCHAND, G., 2005 - Les occupations mésolithiques à l'intérieur du Finistère. Bilan archéographique et méthodologique (20012003), Revue archéologique de l'Ouest, 22, p. 25-84.

Marchand, G., Gallais, J.-Y. et Mens, E., 1998 - Les industries à microlithes entre Loire et Vilaine : bilan et nouvelles perspectives de recherches, Revue archéologique de l'Ouest, 15, p. 15-28.

Marchand, G., Blanchet, S., Chevalier, G., Gallais, J.-Y., Le Goffic, M., Naudinot, N. et Yven, E., 2004 - La fin du Tardiglaciaire sur le Massif armoricain : territoire et culture matérielle, Paléo, 16, p. 137-170. 
Olivier, L., Wirtz, B. et Triboulot, B., 2000 - Assemblages funéraires et territoires dans le domaine hallstattien occidental, in Garcia, D. et Verdin, F. (dir.), Territoires celtiques: espace ethnique et territoires des agglomérations protohistoriques d'Europe occidentale, (Actes du XXIV colloque international de l'AFEAF, Martigues 2000), p. 338-362.

Pétrequin, A.-M. et Pétrequin, P., 1990 - Flèches de chasse, flèches de guerre. Le cas des Danis d'Irian Jaya (Indonésie), Bulletin de la Société préhistorique française, 87, p. 484-511.

Pirazzol, P.-A.,1991 - World atlas of Holocene sea-level changes, Elsevier (Oceanography series, 38), 300 p.

Roussot-Larroque, J., 2000 - Sauveterrien et sauveterriens. Préhistoire du Sud-Ouest, 7-2, p. 159-178.

Rozoy, J.-G., 1978a - Typologie de l'Épipaléolithique (Mésolithique) franco-belge, Bulletin de la Société archéologique champenoise, $\mathrm{n}^{\circ}$ spécial, $121 \mathrm{p}$.

Rozoy, J.-G., 1978b - Les derniers chasseurs. L'Épipaléolithique en France et en Belgique, Bulletin de la Société archéologique champenoise, $\mathrm{n}^{\circ}$ spécial, 605 p, 259 pl.

Rozoy, J.-G., 1997 - Territoires sociaux et environnement en France du nord et Belgique, de 14000 à 6000 BP, in Fagnart, J.- P. et Thévenin, A. (dir.), Le Tardiglaciaire en Europe du Nord-Ouest, (Actes du $119^{\mathrm{e}}$ Congrès national des Sociétés historiques et scientifiques, Amiens 1994), Paris, CTHS, p. 429-454.

Soler Garcia, J. M., 1982 - Los microlitos geometricos. Ensayo de clasificacion tipologica, Helike, 1 (Elche, Universidad Nacional de Educacion a Distancia), p. 117-120.
Thévenin, A., 1991 - Du Dryas III au début de l'Atlantique : pour une approche méthodologique des industries et des territoires dans l'est de la France. $2^{\mathrm{e}}$ partie, Revue archéologique de l'Est et du Centre-Est, 42-1, p. 3-62.

Thévenin, A., 1999 - L'Épipaléolithique et le Mésolithique en France et les régions voisines, in Bintz, P. (dir.), L'Europe des derniers chasseurs; peuplement et paléoenvironnement de l'Épipaléolithique et du Mésolithique, (Actes du $5^{\mathrm{e}}$ colloque international UISP, Grenoble 1995), Paris, CTHS p. 17-24.

Thévenin, A., 2005 - L'espace culturel "méditerranéen ": ses expansions de la fin du Tardiglaciaire au début du Postglaciaire, in Jaubert, J. et Barbaza, M. (dir.), Territoires, déplacements, mobilité, échanges durant la Préhistoire, Actes du 126 e congrès national des sociétés historiques et scientifiques, Toulouse 2001, Paris, CTHS, p. 429-451.

Valdeyron, N., 1994 - Le Sauveterrien, culture et sociétés mésolithiques dans la France du sud durant les $X^{e}$ et IXe millénaires BP, (Thèse de doctorat, Université Toulouse-Le Mirail), 584 p.

Yven, E., 2004 - Approche spatiale et territoriale des industries lithiques. Constantes et variantes dans l'occupation du substrat géographique et la gestion des matières premières lithiques au Mésolithique en Bretagne, (Thèse de doctorat, Université de Bretagne occidentale, Brest), 729 p. 